\title{
Tetracycline Antibiotics in Malaria
}

\author{
Sébastien Briolant, Thierry Fusaï, Christophe Rogier and Bruno Pradines*
}

Unité de recherche en Biologie et Epidémiologie Parasitaires, Institut de Médecine Tropicale du Service de Santé des Armées, et Unité de Recherche sur les Maladies Infectieuses et Tropicales Emergentes - Unité mixte de recherche 6236, Marseille, France

\begin{abstract}
The emergence and rapid extension of Plasmodium falciparum resistance to various antimalarial compounds has gradually limited malaria therapeutic possibilities available to clinicians and the choice of an adapted prophylaxis to travellers specific for their destinations. In this context, doxycycline constitutes an interesting alternative apart from its counter-indications, occurring primarily in children less than eight years old and in pregnant women. Already used successfully in the treatment of malaria in association with quinine in zones of multi-resistances, doxycycline has proven to be effective and well-tolerated in the prevention of malaria. Resistance to doxycycline has not been described until now. The listed prophylactic failures are primarily dependent on an incorrect observation. The mechanisms of action of doxycycline on the parasite are not completely elucidated. The identification of the molecular targets of doxycycline would allow the design of structural analogues that are more active and stable.
\end{abstract}

Key Words: Malaria, doxycycline, prophylaxis, treatment, antimalarial, antibiotics.

\section{INTRODUCTION}

The programme of malaria eradication, initiated in 1960 by the World Health Organization, failed in stable malaria areas, and the current situation remains alarming. Nearly $40 \%$ of the world population lives in endemic areas, with 300 to 500 million new infections and 1.5 to 2.7 million deaths occurring each year [1]. The vector control came up against the appearance of resistances in Anopheles to principal insecticides employed [2]. The vaccine prospects remain limited by the complexity of the biology of Plasmodium falciparum, its antigenic diversity, as well as the inconclusive clinical trials for vaccine candidates in terms of protection and duration of action [3]. The disease prevention and chemotherapy remain a major research focus in the antimalarial fight, and new molecules are constantly required to combat ever-emerging parasitic strains resistant to antimalarial compounds [4].

Chloroquine was introduced in 1940 and has played a dominant role in the chemoprophylaxis and the therapy of malaria. However, extension of chloroquine-resistance to the malaria endemic areas limits its use today [5]. The mechanisms of resistance of $P$. falciparum and the associated molecular markers are generally well-documented for the following antimalarial compounds [6]: chloroquine, mefloquine, halofantrine, cycloguanil, pyrimethamine, sulfadoxine and atovaquone. Cases of resistance to artemisinine and its derivatives used in association in the therapy of malaria have previously been described [7], with some isolates presenting

\footnotetext{
*Address correspondence to this author at the Unité de Recherche en Biologie et Epidémiologie Parasitaires, Institut de Médecine Tropicale du Service de Santé des Armées, Boulevard Charles Livon, Parc du Pharo, BP 46, Pharo, 13998 Marseille Armées, France; Tel: +33 4911501 10; Fax: + 33 4911501 64; E-mail: bruno.pradines@free.fr
}

decreased susceptibilities in vitro to arthemeter and artesunate [8-10]. The recent introduction of Malarone ${ }^{\circledR}$ (association of atovaquone-proguanil) into the chemoprophylaxis of malaria has already fallen victim to the appearance of resistances [11]. Some antibiotics used in the treatment or the prophylaxis of malaria include the macrolides and, in particular, azithromycin, which has been shown to be effective both in vitro [12] and clinically [13].

The tetracyclines, discovered in the beginning of 1940 , are broad-spectrum antibiotics and have a wide range of potency on a number of bacteria, particularly intracellular bacteria, or protozoa such as Plasmodium [14] as well as lymphatic filariasis [15]. The first clinical use of tetracyclines (aureomycin or chlortetracycline) in the treatment of malaria occurred in 1950 [16]. In Mexico, in 1952 [17] and 1956 [18], Ruiz-Sanchez et al. successfully used terramycin (oxytetracycline) in the treatment of uncomplicated malaria to $P$. falciparum and $P$. vivax on a small series of patients (15 and 17, respectively). After the development of resistance to chloroquine in 1960, several studies carried out in $1970[19,20]$ supported the recommendations of the Center for Disease Control for using doxycycline in chemoprophylaxis of $P$. falciparum malaria. Currently, this molecule is used therapeutically in combination with quinine and in chemoprophylaxis in zones of multi-resistances, such as Southeast Asia. Lastly, doxycycline has been used as an initial therapy in chemoprophylaxis in chloroquine-resistance areas by the French military forces deployed in endemic malaria areas.

This review aims to present the pharmacological properties, the mechanisms of action and the activity on the parasite, the therapeutic and chemoprophylactic efficacy, the potential resistance, the tolerability, and the prospects for doxycycline in the treatment of malaria. 


\section{PHARMACOLOGICAL PROPERTIES}

Principal data concerning the pharmacokinetic parameters of doxycycline are summarized in Table 1. Doxycycline is very quickly absorbed per os since it is detectable in blood 15 to 30 minutes after its uptake [21-23]. The majority of absorption is carried out in the duodenum [23] and is not modified by food, dairy products or cations. Conversely, didanosine, iron salts, gastro-intestinal topics, enzymatic inductive anticonvulsivants, alcohol and denutrition decrease its absorption. The bioavailability of doxycycline is nearly $95 \%$. The maximum plasmatic concentration of doxycycline (Cmax) varies from 1.5 to $7 \mu \mathrm{g} / \mathrm{ml}$ and is usually reached in 3 hours, while its half-life varies from 14 to 26 hours [24]. Cmax values are higher in older people due to a reduced digestive elimination and are lower in teenagers for unknown reasons [25]. As much as $93 \%$ of doxycycline is associated with plasmatic proteins, and little accumulates in red blood cells where its concentration is 2.3 times higher than the corresponding culture medium [26]. Doxycycline has good tissue diffusion because of its high liposolubility and is not metabolized. Forty percent of doxycycline is excreted in three days in faeces by bile, and $30 \%$ in the urine.

Several studies were performed to determine the pharmacokinetic properties of doxycycline in healthy volunteers, but little was completed during infections. Only one study was carried out during the treatment of uncomplicated malaria in combination with quinine or artesunate [27]. In a series of 17 patients, the posology of $200 \mathrm{mg}$ per day chosen empirically was determined to be too weak. The authors recommend an initial dose of $400 \mathrm{mg}$ and a twice-daily administration of $200 \mathrm{mg}$ doxycycline in order to maintain plasmatic concentrations at therapeutic rates during the treatment of malaria.

\section{MECHANISMS OF ACTION}

Cyclines are a family of antibiotics long known to inhibit protein synthesis of bacteria. Their mechanism of action was elucidated further at the molecular level when it was determined that they were fixed at the proteins S4, S7 and S9 of the small ribosomal subunit $30 \mathrm{~S}$ and with various ribonucleic acids of the ribosomal RNA 16S [28-31], preventing the binding of aminoacyl transfer RNA to site A of the ribosome, thus blocking the elongation of the translation. However, these mechanisms of action for Plasmodium are much less clearly identified.

In 1976, a study [32] highlighted an inhibition of more than $95 \%$ of the protein synthesis by chlortetracycline on a cytosolic translation system in vitro of Plasmodium knowlesi (simian species of Plasmodium are also responsible for rare

Table 1. Pharmacokinetic Data of Doxycycline

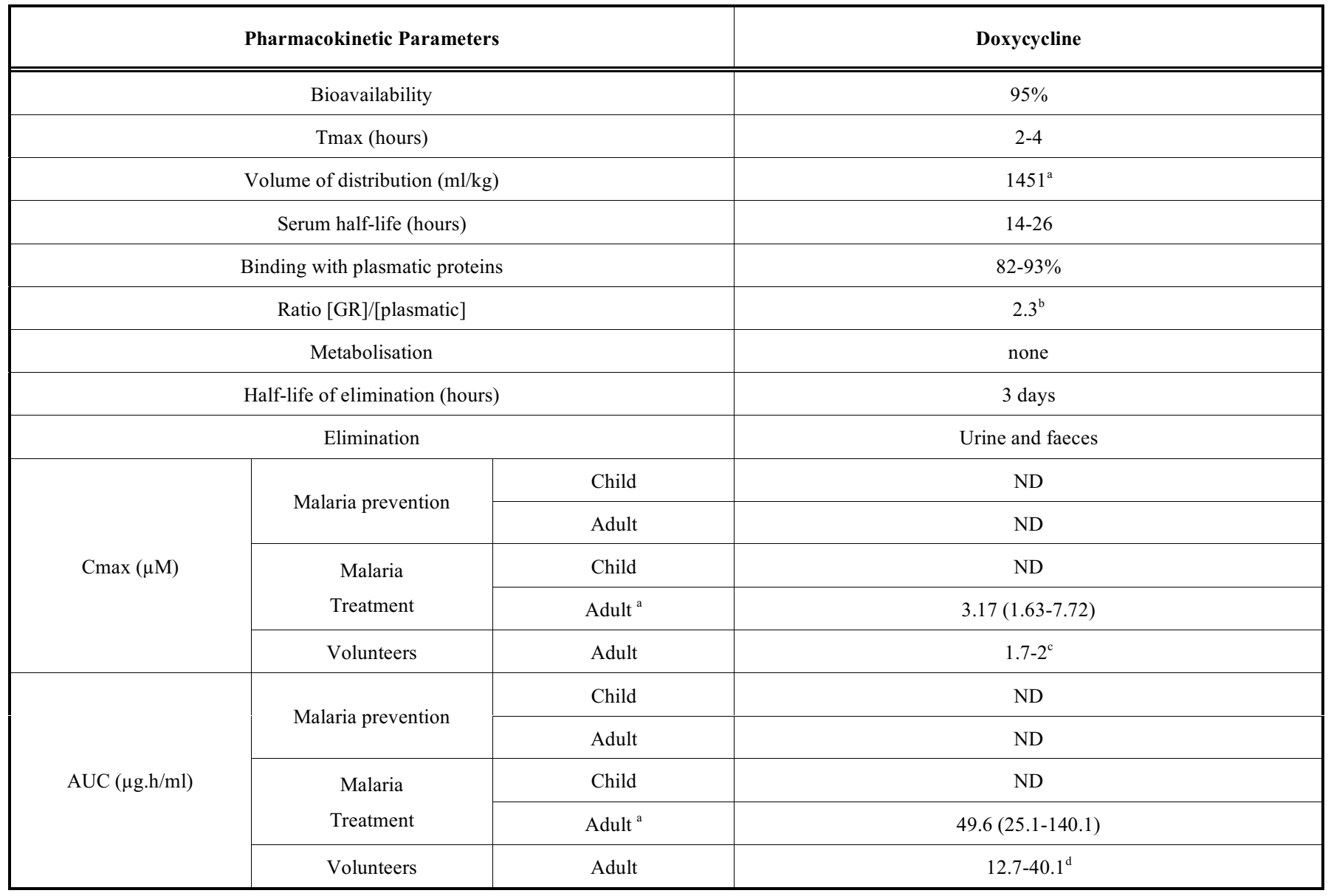

ND: no data, variable ${ }^{\mathrm{a}}$ : according to the weight and the age of the patient (n=17 patient, Newton, 2005), ${ }^{\mathrm{b}}$ : measurements carried out in vitro [26], ${ }^{\mathrm{c}}: \mathrm{Cmax}(\mathrm{mg} / \mathrm{l})$ with $100 \mathrm{mg}$ of doxycycline per os [22], $:$ : AUC (mg/L.h).

$\mathrm{Cmax}=$ peak plasma concentration, Tmax $=$ time to $\mathrm{Cmax}$ and $\mathrm{AUC}=$ area under plasma concentration/time curve 
human infections in Borneo). However, the concentration used $\left(10^{-4} \mathrm{M}\right)$ was more than 10 times the dose used in therapies, and would probably be toxic in humans. Several later studies showed an action of the cyclines on the plasmodial mitochondrion at concentrations of $1 \mu \mathrm{M}$ to $10 \mu \mathrm{M}$, which was similar to clinical concentrations. A study highlighted a synergy of in vitro action between the exposure time of the parasite to the tetracyclines and the increase in the oxygen content of the environment of Plasmodium [33], suggesting an action of the tetracyclines on mitochondrion. The latter is implied in the control of the oxidative stress and in the energy production of anaerobic Plasmodium [34]. According to three previous studies, cyclines would directly inhibit the mitochondrial protein synthesis [35-37], and would also decrease the activity of an enzyme, resulting in the dihydroorotate dehydrogenase being applied in the synthesis of de novo pyrimidines [38]. Doxycycline would inhibit the synthesis of nucleotides and the deoxynucleotides at $P$. falciparum [39], at a concentration much higher than those observed in vivo $(200 \mu \mathrm{M})$. The in vitro exposure of $P$. falciparum to minocycline would also decrease the transcription of mitochondrial genes (subunit I of the cytochrome $\mathrm{C}$ oxidase and the apocytochrome b) and of plastid genes (subunit $\mathrm{rpoB} / \mathrm{C}$ of the RNA polymerase), suggesting an activity on these two organelles [40]. One study showed that doxycycline would act specifically on the apicoplast of $P$. falciparum [41], and to a lesser extent, on the mitochondrion whose division is inhibited at the end of the cycle that the authors allot to the apicoplastic attack (the two organelles having common metabolic pathways). A parasite exposed to $1 \mu \mathrm{M}$ of doxycycline for 20 hours presents during the following cycle (at 72 hours) an inhibition of the apicoplastic replication visualized by fluorescence confocale microscopy, electron microscopy and analysis of the parasitic transcriptome. Two recent studies confirm the specific action of the cyclines on the apicoplast of $P$. falciparum $[42,43]$. However, another team did not note the inhibition of the plastid replication of different Toxoplasma gondii, a member of the Apicomplexan like $P$. falciparum, by submitting the parasite to $100 \mu \mathrm{M}$ of tetracycline for 48 hours [44].

\section{ANTIPLASMODIAL ACTIVITY}

\section{Activity on Sporogonie}

The lack of an in vivo effect of the tetracyclines on the development of gametocytes (suggested by Ruiz Sanchez $[17,18])$ is confirmed by a study performed in 1971 in the United States on healthy volunteers experimentally infected with $P$. falciparum or $P$. vivax [19], and treated by tetracycline or doxycycline. These molecules do not have any action on sporogonie in Anopheles, as they do not decrease the capacity of mosquitoes to become infected after blood feeding on carriers of gametocytes under treatment [20].

\section{Activity on the Hepatic Forms}

In 1972, Willerson et al. showed the following effect of minocycline on the hepatic stages of $P$. falciparum: the administration of $100 \mathrm{mg}$ per day for 7 days, beginning one day prior to exposure to mosquito transmitted sporozoites, prevented malaria in four non-immune healthy volunteers [20]. Several studies performed in vivo on simian models (monkeys rhesus and chimpanzees) infected by P. cynomolgi bas- tianellii, $P$. vivax or $P$. cynomolgi ceylonensis showed that terramycin, minocycline or demeclocycline also had an activity on the hepatic forms [45-47]. In a murine model, doxycycline proves to also be effective on the hepatic stages of $P$. berghei and $P$. yoelii yoelii [48], as the administration of $1.4 \mathrm{mg}$ of doxycycline simultaneously or three hours after the injection of sporozoïtes prevented the appearance of a parasitaemia in $100 \%$ of the rodents $(n=10)$, while the untreated controls became infected.

However, a study carried out in the United States by the American army on non-immune healthy volunteers in 1994 [49] showed that doxycycline was only partially effective on the hepatic forms of $P$. falciparum. Of the twelve subjects who received $100 \mathrm{mg}$ of doxycycline per day three days prior to an exposure to infected mosquitoes and during the six following days, four developed malaria. Moreover, the regular uptake of doxycycline did not alter the level of antibodies against preerythrocytic stages of $P$. falciparum [50]. These results justified the recommendation of the prophylactic schedule currently adopted with doxycycline, which imposes a daily dose of $100 \mathrm{mg}$ and its continuation during four weeks after the return from endemic areas.

\section{Activity on the Erythrocytic Forms}

According to Geary et al. [51], the cyclines are equally active against the three asexual stages of blood development of $P$. falciparum. According to Dahl et al. [41], the old trophozoïtes and the young schizontes would be more susceptible to doxycycline than the young trophozoïtes and the old schizontes. There is a relationship between the amount and duration of exposure and the effect of doxycycline on the erythrocytic stages with an increased activity at the time of the second cycle, even after a short exposure to $1 \mu \mathrm{M}$ during the first cycle (personal data not published). This action against the progeny of treated parasites rather than the parasites exposed to the drugs was termed 'delayed death'.

The in vitro susceptibility of $P$. falciparum to doxycycline estimated by the inhibiting concentration $50 \%\left(\mathrm{IC}_{50}\right)$ is about $5.1 \mu \mathrm{M}$ [3.10-8.38] for multiresistant isolates (from Africa or Southeast Asia) and of $4.3 \mu \mathrm{M}$ [2.90-6.38] for isolates from West Africa [52]. If we compare the values of the $\mathrm{IC}_{50}$ of doxycycline with those of the other antimalarial compounds that are below $1 \mu \mathrm{M}$, doxycycline appears to be much less active. A more recent study carried out on 71 Senegalese isolates (average $\mathrm{IC}_{50}$ of $11.3 \mu \mathrm{M}$ [9.5-13.4] for doxycycline) resulted in a lack of correlation observed between the in vitro estimated $\mathrm{IC}_{50}$ of the isolates to doxycycline and arthemeter, chloroquine, quinine, amodiaquine, pyrimethamine or cycloguanil, suggesting an absence of cross resistance between these molecules [53]. A synergy of action does not exist between the cyclines and chloroquine, mefloquine or quinine [54]. On the other hand, a synergy of action appeared between doxycycline and atovaquone [55, 56] and between doxycycline and artemisinine [57, 58], despite previous studies that showed an additive effect $[59,60]$. This justifies doxycycline therapeutic use in combination with a fast schizontocide. The distribution and range of doxycycline $\mathrm{IC}_{50}$ values were determined for 747 African isolates. A "triple normal" distribution was fitted to the data using a Bayesian mixture modelling approach. The values for all 747 isolates were classified into 3 components: a first 
component $\mathrm{A}$ with an $\mathrm{IC}_{50}$ mean of $4.9 \mu \mathrm{M}( \pm 2.1 \mu \mathrm{M})$, a second component $\mathrm{B}$ with an $\mathrm{IC}_{50}$ mean of $7.7 \mu \mathrm{M}( \pm 1.2$ $\mu \mathrm{M})$, and a third component $\mathrm{C}$ with an $\mathrm{IC}_{50}$ mean of $17.9 \mu \mathrm{M}$ $( \pm 1.4 \mu \mathrm{M})$. The cut-off for reduced susceptibility to doxycycline in vitro was estimated by the geometric mean +2 standard deviations of the $\mathrm{IC}_{50}$ values of the $P$. falciparum isolates associated with $\mathrm{C}$ component, that is to say $34.2 \mu \mathrm{M}$. Isolates with an $\mathrm{IC}_{50}>35 \mu \mathrm{M}$ are considered as isolates with reduced susceptibility to doxycycline in vitro (submitted publication).

\section{EFFICACY OF DOXYCYCLINE}

\section{Malaria Treatment}

All of the studies undertaken in $1950[17,18]$ and 1970 $[19,20,61,62]$ showed the efficacy of the cyclines in monotherapy in the treatment of uncomplicated $P$. falciparum and $P$. vivax malaria. However, in the event of infections with $P$. vivax, some relapses were observed [63] during the two to three months following the first clinical malaria episode or during the first month [64], evidencing an insufficient activity of the cyclines on the hypnozoïtes. Moreover, a radical cure was obtained only after a minimal duration of 7 days treatment of $P$. falciparum (with a dose of $200 \mathrm{mg}$ per day of doxycycline), leading to the disappearance of the parasites in only four to five days on average and approximately 7 days for $P$. vivax. Because of the risk of evolution of uncomplicated $P$. falciparum malaria towards severe forms, the slow schizontocide activity of doxycycline is not suggested for use as cyclines in monotherapy. Conversely, their association with other antimalarial compounds in zones of multiresistances (Southeast Asia and South America) were the subject of many studies [65-80], which are summarized in Table 2.

The "standard" treatment of uncomplicated $P$. falciparum malaria in zones of multi-resistances relies on the association of quinine $(30 \mathrm{mg} / \mathrm{kg} /$ day) to doxycycline (200 $\mathrm{mg}$ per day) for 7 days [81]. This treatment makes it possible to obtain a radical cure even in the event of reduction in the in vitro susceptibility of isolates to quinine [82]. This bitherapy has a therapeutic efficacy from 91 to $100 \%$ in zones of multiresistances. All other associations tested are lower or equal, in terms of therapeutic efficacy, and are often more expensive, except the combination clindamycin-quinine, which would constitute an interesting alternative in the treatment of malaria in pregnant women and children aged less than 8 years to which the cyclines are counter-indicated.

\section{Malaria Chemoprophylaxis}

Currently, doxycycline is used in malaria chemoprophylaxis at $100 \mathrm{mg}$ per day from the first day in endemic areas up to four weeks after return. This schedule, initially recommended by the WHO in 1985, was based on previous studies $[19,20]$. The principal studies relating to the efficacy and the tolerance of doxycycline in malaria prevention are summarized in Table 3 . These studies were carried out on the following various populations followed for periods of at least 28 days after the halt of prophylaxis: semi-immune or immune subjects living in endemic areas [83-90] and nonimmune travellers, mainly soldiers [91-97]. The results show efficacy from 91 to $99 \%$ in immune and semi-immune sub- jects, and from 95 to $100 \%$ in travellers. The majority of the failures observed in the malaria prophylaxis to $P$. falciparum were attributed to a maladjustment of posology confirmed by weak plasmatic concentrations in doxycycline [87], to the use of half dose [84] or to poor observance [92, 98]. However, true prophylactic failures exist. For example, two Australian soldiers presented a $P$. falciparum malaria two weeks after their return from New Guinea in spite of good observance (testified by plasmatic concentration of doxycycline) [93]. No in vitro chemosusceptibility test of the isolates to doxycycline was performed in these cases.

The major clinical studies of efficacy and tolerance of doxycycline in malaria prophylaxis against $P$. vivax are summarized in Table 4. The results show efficacy from 83.1 to $98.7 \%$ in immune and semi-immune subjects, and from 52.7 to $100 \%$ in travellers. Doxycycline has poor efficacy in the prevention of $P$. vivax malaria relapses.

\section{Resistance to Doxycycline}

The mechanisms of resistance of bacteria to the cyclines and the implicated proteins were previously identified [14] and are summarized in Table $\mathbf{5}$.

Efflux pumps were the most studied among the Tet proteins, encoded by genes belonging to the major facilitator superfamily, and include over 300 individual proteins [99]. All the tet efflux protein genes code membrane-associated proteins that export tetracycline from the cell. Export of tetracycline reduces the intracellular drug concentration and thus protects the ribosome within the cell. Recently, nucleotide sequence and transfer properties of two novel types of Actinobacillus pleuropneumoniae plasmids carrying the tetracyclines resistance gene $\operatorname{tet}(\mathrm{H})$ were discovered [100]. The weak accumulation of tetracycline inside the parasite [26], in spite of sequences homology between a putative transporter of $P$. falciparum and bacterial efflux pumps (Fig. 1), may suggest the absence of this type of mechanism of resistance to the cyclines in $P$. falciparum (no X-ray crystallographic data are available about efflux pumps in order to predict and compare three-dimensional structures).

The tet $\mathrm{X}$ genes have been identified in anaerobic bacteria of the genus Bacteroides. TetX protein is a flavin-dependent monooxygenase conferring resistance to tetracycline antibiotics by degradation in vitro and in vivo [101]. No homology of sequence was found between the tet $\mathrm{X}$ gene and any gene of the genome of $P$. falciparum.

The existence of specific mutations in bacterial rRNA $16 \mathrm{~S}$ gene can confer a resistance to tetracyclines to Helicobacter pylori by diminution of drug fixing to ribosomes [102].

Ribosomal protection proteins are cytoplasmic proteins that protect ribosomes from the action of tetracycline in a GTP-dependent way [103, 104], and display sequence similarity to translation elongation factors EF-G/EF-2 and EF$\mathrm{Tu} / \mathrm{EF}-1 \alpha$ [105]. Likewise, they possess four functional domains EFTu, EFTu D2, EFG-IV and EFG-C. Interestingly, P. falciparum possesses a tetQ GTPase family gene (PFL$1710 \mathrm{c}$ number access in Plasmodb database). The amino acid sequences of five bacterial protection proteins and the $P$. falciparum tetQ GTPase protein were aligned using the 
Table 2. Clinical Studies Comparing the Association with Cyclines and other Therapeutics in the Treatment of $P$. falciparum Malaria

\begin{tabular}{|c|c|c|c|c|c|c|c|}
\hline \multicolumn{3}{|c|}{ Study } & \multirow{2}{*}{$\begin{array}{c}\text { Treatment } \\
\text { QT } \\
\text { QCq }\end{array}$} & \multirow{2}{*}{$\begin{array}{c}\begin{array}{c}\text { No. of } \\
\text { Patients }\end{array} \\
30 \\
36\end{array}$} & \multirow{2}{*}{$\begin{array}{c}\begin{array}{c}\text { Parasitic } \\
\text { Clearance } \\
\text { (Days) }\end{array} \\
3.6 \\
3.2\end{array}$} & \multirow{2}{*}{$\begin{array}{c}\begin{array}{c}\text { Disappear- } \\
\text { ance of the } \\
\text { Fever (Days) }\end{array} \\
\text { ND } \\
\text { ND }\end{array}$} & \multirow{2}{*}{$\begin{array}{c}\text { Efficacy (\%) } \\
\text { (Duration of the } \\
\text { Follow-Up in Days) } \\
96.6(28) \\
41.6^{\mathrm{a}}(28)\end{array}$} \\
\hline Colwell, 1972 & Adults & Thailand & & & & & \\
\hline Chin, 1973 & Adults & Thailand & $\begin{array}{c}\mathrm{Q}^{\mathrm{b}} \\
\mathbf{Q T}^{\mathrm{b}} \\
\mathbf{Q T P y}^{\mathbf{b}}\end{array}$ & $\begin{array}{l}10 \\
12 \\
13\end{array}$ & $\begin{array}{l}2.1 \\
2.4 \\
1.6\end{array}$ & $\begin{array}{c}3 \\
2.2 \\
2.8\end{array}$ & $\begin{array}{c}75(28) \\
66.7(28) \\
66.7(28)\end{array}$ \\
\hline Colwell, 1973 & Adults & Thailand & $\begin{array}{l}\mathbf{Q T}^{\mathrm{c}} \\
\mathrm{QB}^{\mathrm{c}}\end{array}$ & $\begin{array}{l}32 \\
31\end{array}$ & $\begin{array}{l}2.4 \\
2.7\end{array}$ & $\begin{array}{l}4 \\
3\end{array}$ & $\begin{array}{l}84(28) \\
81(28)\end{array}$ \\
\hline $\begin{array}{c}\text { Noeypatimanond, } \\
1983\end{array}$ & Adults Children & Thailand & TAm & 51 & 4.1 & ND & $96(28)$ \\
\hline Giboda, 1988 & Adults & Kampuchea & $\begin{array}{c}\text { Q } \\
\text { QT }\end{array}$ & $\begin{array}{l}43 \\
22\end{array}$ & $\begin{array}{l}5.6 \\
5.9\end{array}$ & $\begin{array}{l}3.6 \\
3.8\end{array}$ & $\begin{array}{l}41.8^{\mathrm{d}}(12) \\
1 \mathbf{0 0}^{\mathrm{d}}(12)\end{array}$ \\
\hline $\begin{array}{c}\text { Looareesuwan, } \\
1994\end{array}$ & Adults & Thailand & $\begin{array}{l}\text { MT } \\
\text { QT }\end{array}$ & $\begin{array}{l}47 \\
46\end{array}$ & $\begin{array}{l}2.7 \\
3.1\end{array}$ & $\begin{array}{c}2 \\
2.6\end{array}$ & $\begin{array}{l}94(28) \\
98(28)\end{array}$ \\
\hline $\begin{array}{c}\text { Looareesuwan, } \\
1994\end{array}$ & Adults & Thailand & $\begin{array}{l}\text { MD } \\
\text { AD }\end{array}$ & $\begin{array}{l}48 \\
49\end{array}$ & $\begin{array}{l}2.9 \\
2.7\end{array}$ & $\begin{array}{l}1.7 \\
1.6\end{array}$ & $\begin{array}{l}\mathbf{9 6}(28) \\
\mathbf{8 0}(28)\end{array}$ \\
\hline Metzger, 1995 & Adults & Gabon & $\begin{array}{l}\text { Q } \\
\text { QCl } \\
\text { QD }\end{array}$ & $\begin{array}{l}37 \\
36 \\
35\end{array}$ & $\begin{array}{l}2.2 \\
2.4 \\
2.2\end{array}$ & $\begin{array}{l}2 \\
2 \\
2\end{array}$ & $\begin{array}{l}38(28) \\
92(28) \\
91(28)\end{array}$ \\
\hline $\begin{array}{c}\text { Na-Bangchang, } \\
1996\end{array}$ & Adults & Thailand & $\begin{array}{l}\mathrm{AAz}^{\mathrm{e}} \\
\mathbf{A D}^{\mathrm{e}}\end{array}$ & $\begin{array}{l}30 \\
30\end{array}$ & $\begin{array}{l}1.2 \\
1.3\end{array}$ & $\begin{array}{l}0.8 \\
1.1\end{array}$ & $\begin{array}{l}14.8(28) \\
\mathbf{5 3 . 3}(28)\end{array}$ \\
\hline $\begin{array}{c}\text { Looareesuwan, } \\
1996\end{array}$ & Adults & Thailand & $\begin{array}{r}\text { AtT } \\
\text { AtP } \\
\text { DTA } \\
\text { AtPy }\end{array}$ & $\begin{array}{l}25 \\
24 \\
22 \\
13\end{array}$ & $\begin{array}{c}2.8 \\
3 \\
2.7 \\
2.6\end{array}$ & $\begin{array}{l}1.3 \\
3.5 \\
2.2 \\
2.5\end{array}$ & $\begin{array}{c}100(28) \\
100(28) \\
91(28) \\
77(28)\end{array}$ \\
\hline Duarte, 1996 & Adults Children & Brazil & $\begin{array}{l}A T^{f} \\
Q T^{f}\end{array}$ & $\begin{array}{l}88 \\
88\end{array}$ & $\begin{array}{l}\mathrm{ND} \\
\mathrm{ND}\end{array}$ & $\begin{array}{l}\text { ND } \\
\text { ND }\end{array}$ & $\begin{array}{l}80(28) \\
77(28)\end{array}$ \\
\hline Bunnag, 1996 & Adults & Thailand & $\begin{array}{l}\mathbf{Q T}^{\mathrm{g}} \\
\mathbf{Q T}^{\mathrm{g}}\end{array}$ & $\begin{array}{l}46 \\
40\end{array}$ & $\begin{array}{l}3.7 \\
3.7\end{array}$ & $\begin{array}{l}3.1 \\
3.1\end{array}$ & $\begin{array}{c}\mathbf{8 7}(28) \\
\mathbf{1 0 0}(28)\end{array}$ \\
\hline $\begin{array}{c}\text { Pukrittayakamee, } \\
2000\end{array}$ & Adults Children & Thailand & $\begin{array}{c}\text { Q } \\
\text { QCl } \\
\text { QT }\end{array}$ & $\begin{array}{l}68 \\
68 \\
68\end{array}$ & $\begin{array}{l}3.2 \\
3.3 \\
3.2\end{array}$ & $\begin{array}{c}2.3 \\
2 \\
1.5\end{array}$ & $\begin{array}{c}87(28) \\
100(28) \\
98(28)\end{array}$ \\
\hline Taylor, 2001 & Adults & Irian Jaya & $\begin{array}{c}\text { Cq } \\
\text { D } \\
\text { DCq }\end{array}$ & $\begin{array}{l}30 \\
20 \\
39\end{array}$ & $\begin{array}{l}2.8 \\
3.8 \\
3.4\end{array}$ & $\begin{array}{l}1.7 \\
2.6 \\
2.5\end{array}$ & $\begin{array}{c}22(28) \\
64.7(28) \\
90.9(28)\end{array}$ \\
\hline $\begin{array}{c}\text { Pukrittayakamee, } \\
2004\end{array}$ & Adults Children & Thailand & $\begin{array}{c}\text { Q } \\
\text { QT } \\
\text { QPr } \\
\text { QPr } \\
\text { With } \\
\text { APr }\end{array}$ & $\begin{array}{l}30 \\
30 \\
29 \\
37 \\
23 \\
27\end{array}$ & $\begin{array}{l}3.3 \\
3.4 \\
3.2 \\
3.3 \\
2.9 \\
2.6\end{array}$ & $\begin{array}{c}2.6 \\
1.4 \\
2 \\
2.5 \\
1.4 \\
1.3\end{array}$ & $\begin{array}{c}84(28) \\
\mathbf{1 0 0}(28) \\
72(28) \\
93(28) \\
90.5(28) \\
84(28)\end{array}$ \\
\hline Alecrim, 2006 & Adults & Brazil & $\begin{array}{l}\text { ArLu } \\
\text { QD }\end{array}$ & $\begin{array}{l}28 \\
31\end{array}$ & $\begin{array}{l}1.9 \\
3.3\end{array}$ & $\begin{array}{l}\text { ND } \\
\text { ND }\end{array}$ & $\begin{array}{l}100(6) \\
100(6)\end{array}$ \\
\hline
\end{tabular}

a: study performed in zones of chloroquine-resistance; ${ }^{b}$ : quinine 7 days, quinine tetracycline 3 days and quinine tetracycline pyrimethamine 3 days; ${ }^{c}$ : quinine 1 day, tetracycline 7 days and bactrim 5 days; ${ }^{\mathrm{d}}$ : susceptibility in vivo after 10 days of treatment; ${ }^{\mathrm{e}}$ : artesunate amount of load + doxycycline 5 days or azithromycin 2 days; ${ }^{\mathrm{f}}$ : artesunate + tetracycline 7 days and quinine 3 days + tetracycline 7 days; ${ }^{\mathrm{g}}$ : quinine 5 or 7 days + tetracycline 7 days.

Q: quinine; T: tetracycline; Cq: chloroquine; Py: pyrimethamine; B: bactrim; Am: amodiaquine; M: mefloquine; D: doxycycline; A: artesunate; Cl: clindamycin; Az: azithromycin;

At: atovaquone; P: proguanil; Pr: primaquine; Ar: arthemeter; Lu: lumefantrine. 
Table 3. Clinical Studies of Doxycycline Efficacy and Tolerance, Comparatively in $P$. falciparum Malaria Chemoprophylaxis

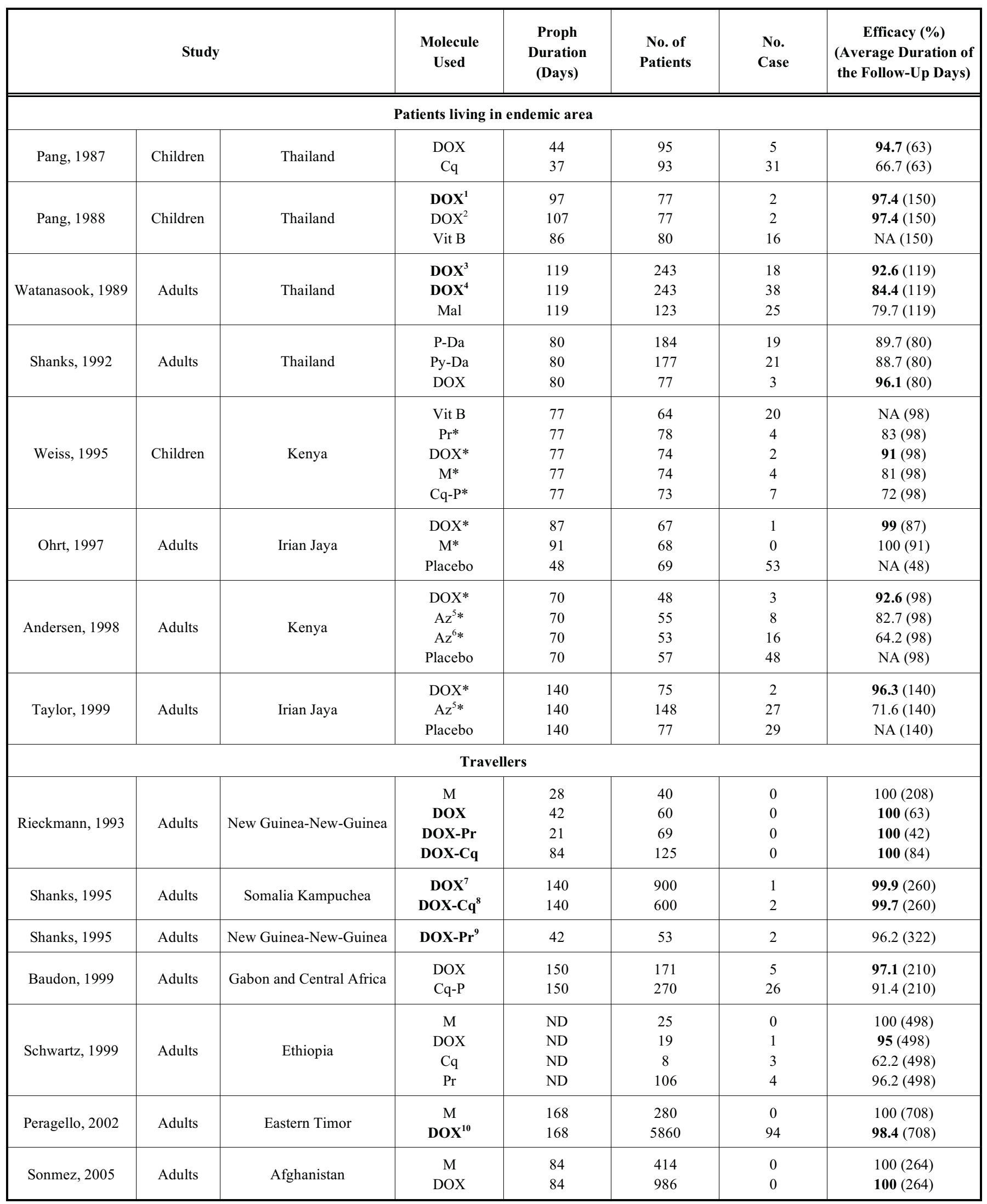

NA: no appreciable; $*$ : efficacy $=100 \mathrm{X}[1$ (rate of failure/rate of failure in the placebo group) $] ;{ }^{1}:$ doxycycline $100 \mathrm{mg} / \mathrm{day}$ or $50 \mathrm{mg} / \mathrm{day}$ according to weight's; ${ }^{2}:$ doxycycline 50 $\mathrm{mg} /$ day or $25 \mathrm{mg} /$ day according to weight's; ${ }^{3}$ : doxycycline $100 \mathrm{mg} /$ day; ${ }^{4}$ : doxycycline $50 \mathrm{mg} / \mathrm{day} ;{ }^{5}$ : azithromycin $250 \mathrm{mg}$ /day; ${ }^{6}$ : azithromycin $1000 \mathrm{mg} / \mathrm{week} ;{ }^{7}:$ + primaquine with the return $15 \mathrm{mg} /$ day 15 days; ${ }^{8}:$ + primaquine with the return $15 \mathrm{mg} /$ day 15 days; ${ }^{9}:$ + primaquine $7.5 \mathrm{mg} /$ day 5 days; ${ }^{10}:+$ primaquine $7.5 \mathrm{mg} 3$ times/day 14 days with the return. DOX: doxycycline; Cq: chloroquine; Mal: Maloprim ${ }^{\circledR}$ (pyrimethamine dapsone); P: proguanil; Py: pyrimethamine; Da: dapsone; Pr: primaquine; M: mefloquine; Az: azithromycin. 
Table 4. Clinical Studies of Doxycycline Efficacy and Tolerance, Compared in P. vivax Malaria Chemoprophylaxis

\begin{tabular}{|c|c|c|c|c|c|c|c|}
\hline \multicolumn{8}{|c|}{ Patients living in endemic area } \\
\hline Pang, 1988 & Children & Thailand & $\begin{array}{l}\text { DOX }^{1} \\
\text { DOX }^{2} \\
\text { Vit B }\end{array}$ & $\begin{array}{c}97 \\
107 \\
86\end{array}$ & $\begin{array}{l}77 \\
77 \\
80\end{array}$ & $\begin{array}{c}3 \\
16 \\
62\end{array}$ & $\begin{array}{l}96.1(150) \\
\mathbf{7 9 . 2}(150) \\
\text { NA (150) }\end{array}$ \\
\hline Watanasook, 1989 & Adults & Thailand & $\begin{array}{c}\text { DOX }^{3} \\
\text { DOX }^{4} \\
\text { Mal }\end{array}$ & $\begin{array}{l}119 \\
119 \\
119\end{array}$ & $\begin{array}{l}243 \\
243 \\
123\end{array}$ & $\begin{array}{l}28 \\
41 \\
64\end{array}$ & $\begin{array}{c}\mathbf{8 8 . 5}(119) \\
\mathbf{8 3 . 1}(119) \\
48(119)\end{array}$ \\
\hline Shanks, 1992 & Adults & Thailand & $\begin{array}{l}\text { P-Da } \\
\text { Py-Da } \\
\text { DOX }\end{array}$ & $\begin{array}{l}80 \\
80 \\
80\end{array}$ & $\begin{array}{c}184 \\
177 \\
77\end{array}$ & $\begin{array}{c}3 \\
22 \\
1\end{array}$ & $\begin{array}{l}98.4(80) \\
87.6(80) \\
98.7(80)\end{array}$ \\
\hline \multicolumn{8}{|c|}{ Travellers } \\
\hline Rieckmann, 1993 & Adults & New Guinea-New-Guinea & $\begin{array}{c}\text { M } \\
\text { DOX } \\
\text { DOX-Pr } \\
\text { DOX-Cq }\end{array}$ & $\begin{array}{l}28 \\
42 \\
21 \\
84\end{array}$ & $\begin{array}{c}40 \\
60 \\
69 \\
125\end{array}$ & $\begin{array}{l}4 \\
2 \\
0 \\
0\end{array}$ & $\begin{array}{c}90(208) \\
96.7(63) \\
100(42) \\
100(84)\end{array}$ \\
\hline Shanks, 1995 & Adults & Somalia Kampuchea & $\begin{array}{c}\text { DOX }^{6} \\
\text { DOX-Cq }^{7}\end{array}$ & $\begin{array}{l}140 \\
140\end{array}$ & $\begin{array}{l}900 \\
600\end{array}$ & $\begin{array}{l}2 \\
6\end{array}$ & $\begin{array}{c}99.8(260) \\
99(320)\end{array}$ \\
\hline Shanks, 1995 & Adults & New Guinea-New-Guinea & DOX-Pr ${ }^{8}$ & 42 & 53 & 9 & $83(322)$ \\
\hline
\end{tabular}

NA: no appreciable; *: efficacy $=100 \mathrm{X}[1$ (rate of failure/rate of failure in the placebo group) $] ; ;^{1}:$ doxycycline $100 \mathrm{mg} / \mathrm{day}$ or $50 \mathrm{mg} / \mathrm{day}$ according to weight's; ${ }^{2}:$ doxycycline 50 $\mathrm{mg} / \mathrm{day}$ or $25 \mathrm{mg} /$ day according to weight's; ${ }^{3}$ : doxycycline $100 \mathrm{mg} /$ day; ${ }^{4}$ : doxycycline $50 \mathrm{mg} / \mathrm{day} ;{ }^{5}$ : azithromycine $250 \mathrm{mg} / \mathrm{day} ;{ }^{6}$ : + primaquine with the return $15 \mathrm{mg} / \mathrm{day} 15 \mathrm{days}$ $7:$ + primaquine with the return $15 \mathrm{mg} /$ day 15 days; ${ }^{8}:+$ primaquine $7.5 \mathrm{mg} /$ day 5 days; ${ }^{9}:$ + primaquine $7.5 \mathrm{mg} 3$ times/day 14 days with the return.

DOX: doxycycline; Cq: chloroquine; Mal: Maloprim ${ }^{\circledR}$ (pyrimethamine dapsone); P: proguanil; Py: pyrimethamine; Da: dapsone; Pr: primaquine; M: mefloquine; Az: azithromycin

Table 5. Mechanisms of Bacterial Resistance Mediated by tet and otr Genes

\begin{tabular}{|c|c|c|c|}
\hline $\begin{array}{c}\operatorname{tet}(\mathrm{A}),(\mathrm{B}),(\mathrm{C}),(\mathrm{D}),(\mathrm{E}) \\
\operatorname{tet}(\mathrm{G}),(\mathrm{H}),(\mathrm{I}),(\mathrm{J}),(\mathrm{K}) \\
\operatorname{tet}(\mathrm{L}),(\mathrm{V}),(\mathrm{Y}),(\mathrm{Z}) \\
\operatorname{tcr} 3 \\
\operatorname{tet}(30),(31) \\
\operatorname{otr}(\mathrm{B}) \\
\operatorname{tet} \mathrm{P}(\mathrm{A})\end{array}$ & $\begin{array}{c}\operatorname{tet}(\mathrm{M}) \\
\operatorname{tet}(\mathrm{O}) \\
\operatorname{tet}(\mathrm{Q}),(\mathrm{S}),(\mathrm{T}) \\
\operatorname{tet}(\mathrm{W}) \\
\text { tet } \\
\operatorname{otr}(\mathrm{A}) \\
\operatorname{tet} \mathrm{P}(\mathrm{B})\end{array}$ & $\operatorname{tet}(\mathrm{X})$ & $\begin{array}{l}\operatorname{tet}(\mathrm{U}) \\
\operatorname{otr}(\mathrm{C})\end{array}$ \\
\hline
\end{tabular}

multiple-sequence alignment program ClustalW [106]. As shown in Fig. (2), there is a great homology or identity in the sequences and the three-dimensional structures of these pro- teins (Figs. 3, $\mathbf{4}$ and 5). P. falciparum may have the capacity to resist tetracycline in this manner. 


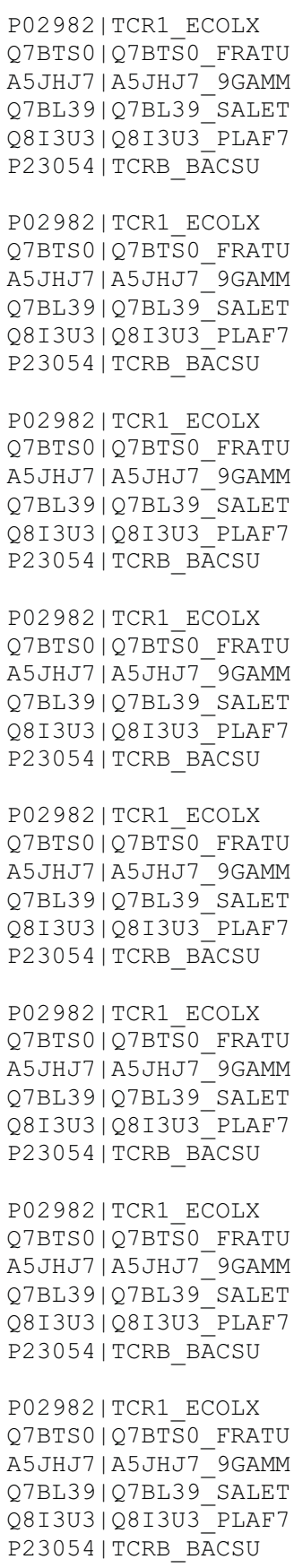

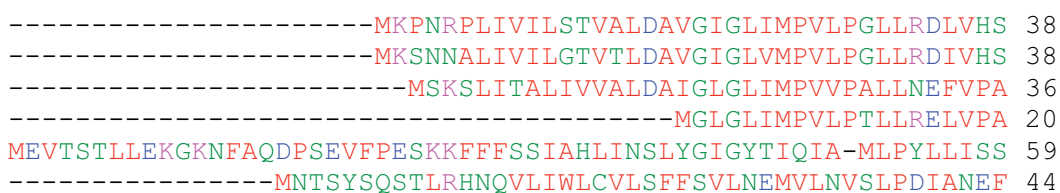

NDVTAHYGILIALYALMOFACAPVLGALSDRFGRRPVLIVSLAGAAVDYAIMATAPFLW- 97 DS IASHYGVLLALYALMQFLCAPVLGALSDRFGRRPVLLASLLGATIDYAIMATTPVLW- 97 EQTAFHYGVFLSLYAFMQVFCAPVLGRLSDRYGRRI ILLVSFLGATIDYS IMAAAPVLW- 95 EOVAGHYGALLSLYALMOVVFAPMLGOLSDSYGRRPVLLASLAGAAVDYTIMASAPVLW- 79 NAGIEHNGYLLTLFSLLQFTGS IFFGRMADIWGVKKSFYLSLISSCLMYLMIMVCESTW- 118 NKLPASANWVNTAFMLTFS IGTALYGKLSDOLGIKNLLLFGIMVNGLGS I IGFVGHSFFP 104

VLYIGRIVAGITGATG-AVAGAYIADITDGDERARHFGFMSACFGFGMVAGPVLGGLMGG 156 ILYAGRIVAGITGATG-AVAGAYIADITDGEDRARHFGLMSACFGVGMVAGPVAGGLLGA 156 VLYIGRIISGVTGATG-AIAASIIADTTKOEERARWFGFMGACFGAGMIAGPAIGGVLGD 154 VLYIGRLVSGVTGATG-AVAASTIADSTGEGSRARWFGYMGACYGAGMIAGPALGGMLGG 138 AYYIS-FLPSFFMQTF-QASSLIVCLKTNFDKRTGALGYLNLSYGMGIIFGSFLAGVMVN 176 ILILARFIOGIGAAAFPALVMVVVARYIPKENRGKAFGLIGSLVAMGEGVGPAIGGMVAH 164

FS--PHAPFFAAAALNGLNFLTGCFLLPESHKGERRPLRREALNP----LASFRWARGMT 210 IS--LHAPFLAAAVLNGLNLLIGCFLMQESHKGERRPMPLRAFNP----VSSFRWARGMT 210 IS--VHAPFVAGALINAIAFCLVAFLLPKTP-SQPPEGQPAKINL----FEGFRFNFAVQ 207 IS--AHAPFIAAALLNGFAFLLACIFLKETHHSHGGTGKPVRIKP----FVLLRLDDALR 192 FVG-SRGNLLIALLSQLIALCISTTLEEDPKLLKSSNVDKMKMSE----ILLSIKNEYIR 231 YIHWSYLLIIPTATIITVPFLIKLLKKEERIRGHIDMAGIILMSAGIVFFMLFTTSYRES 224

VVAALMAVF--FIMQLVGQVP------------------------AALWVIFGE---- 238

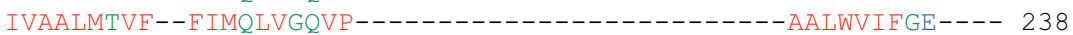

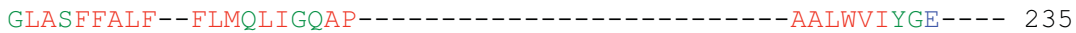

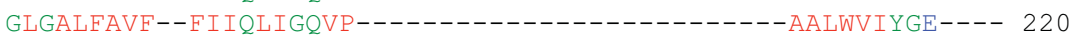
VLNLFKKTYGICLLILFGLIP_-_-_-_-_-_-_-_-_-_-_----ILMTKFAFAPVVV 265 FLIISILAFFIFVQHIRKAQDPFVDPELGKNVFFVIGTLCGGLIFGTVAGFVSMVPYMMK 284

DRFHWDATTIGISLAAFGILHSLAQAMITGPVAARLGERRALMLGMIADGTGYILLAFA- 297 DRFRWSATMIGLSLAVFGILHALAQAFVTGPATKRFGEKQAIIAGMAADALGYVLLAFA- 297 QRLNWDIGTAGVSLAVFGAAHTFVQAVLTGTLSKRLGDRGVLLLGMGADMCGFLLLAFI- 294 DRFQWNTATVGLSLAAFGATHAIFQAFVTGPLSSRLGERRTLLFGMAADATGFVLLAFA- 279 DMFKLTPSHTSYLMTYAGITIIAEGILAPYLSSLLGDMICCKYSIPLTLTGFLLLSLCG 325 DVHHLSTAAIGSGIIFPGTMSVIIFGYIGGLLVDRKGSLYVLTIGSALLSSGFLIAAFFI 344

-TRGWMAFPIMVLIASGGIGMPALQAMLSRQVDEERQGQLQGSLAALTSLTSIVGP-LLF 355 -TRGWMAFPIMILLASGGIGMPALQAMLSRQVDDDHQGQLQGSLAALTSLTSITGP-LIV 355 -TQSWMVLPAIFMLATGG I GMPALQAI ISGLVCDEKQGALQGTLTGLTNITSIIGP-VGF 352 -TOGWMVFPILLILAAGGVGMPALOAMLSNNVSSNKOGALOGTLTSITNLSSIAGP-LGF 337 ANESLVLIFMS IPLCGGALIY ICGTSQMTKRVEESELGSIIGLNTSLFYAVTI IAPYIAF 385 DAAPWIMTIIVIFVFGGLSFTKTVISTVVSSSLKEKEAGAGMSLINFTSFLSEGTGIAIV 404

TAIYAASITTWNGWAWIAGAALYLLCLPALRRGLWSGAGORADR--.---.---- 399 TAIYAASASTWNGLAWIVGAALYLVCLPALRRGAWSRATST-------------- 396

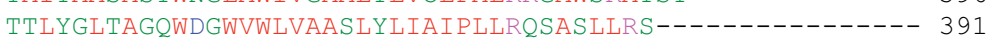
TALYSATAGAWNGWVWIVGAILYLICLPILRRPFATSL---------------- 375 KSYIALGLGLYWLLCAFICEVVTFYIFVLDKSTLKIFKDDKDSIETMFSSIKS IL 440 GGLLSIGELDHRLIPIDVDHSTYLYSNMLILFAGIIVICWLVILNVYKRSRRHG- 458

The Swiss-prot accession numbers P02982, P23054, Q7BTS0, Q7BL39, A5JHJ7 and Q8I3U3 correspond respectively to tetA (Escherichia coli), tetB (Bacillus subtilis), tetC (Francisella tularensis), tetG (Salmonella enterica), tetY (Aeromonas bestiarum) and a putative transporter (Plasmodium falciparum 3D7). Residues are coloured according to their physical and chemical properties: hydrophobic $=$ red, $b a s i c=$ pink, acidic $=$ blue, hydrophilic $=$ green .

\section{Fig. (1). CLUSTALW alignment of five bacterial efflux pumps protein sequences and a putative transporter of Plasmodium falcipa-} rum.

Drug pressure with cyclines was performed in a murine model in P. berghei [107]. The administration of increasing minocycline doses to mice infected with $10^{7}$ parasites after 86 successive passages over a 600 -day period made it possible to obtain a "resistant" strain with $\mathrm{IC}_{50}=600 \mathrm{mg} / \mathrm{kg} /$ day only six times higher than that of the susceptible starting strain $(100 \mathrm{mg} / \mathrm{kg} /$ day $)$. However, this resistance was unsta- ble. After 16 additional passages without drug pressure in mice, the strain returned to its initial susceptibility level.

In spite of prophylactic failures with doxycycline, no resistance was observed in in vitro chemosusceptibility test. To date, there is no molecular marker associated with $P$. falciparum resistance to doxycycline. In addition, the therapeutic failures reported with association quinine-doxycycline 
CAA52967 Q48791

CAA69103

CAD20560

AAF01499

AAN36428

CAA52967

Q48791

CAA69103

CAD20560

AAF01499

AAN36428

CAA52967

Q48791

CAA69103

CAD20560

AAF01499

AAN36428

CAA52967

Q48791

CAA69103

CAD2 0560

AAF01499

AAN 36428

CAA52967

Q48791

CAA69103

CAD20560

AAF01499

AAN36428

CAA 52967

Q48791

CAA69103

CAD20560

AAF01499

AAN3 6428

CAA52967

Q48791

CAA69103

CAD2 0560

AAF01499

AAN36428

CAA52967

Q48791

CAA69103

CAD20560

AAF01499

AAN36428

CAA52967

Q48791

CAA69103

CAD20560

AAF01499

AAN 36428

CAA52967

Q48791

CAA69103

CAD20560

AAF01499

AAN 36428

CAA52967

Q48791

CAA69103

CAD20560

AAF01499

\begin{abstract}
------------------MKIINIGVLAHVDAGKTTLTESLIYNSGAITELGSVDKG 39

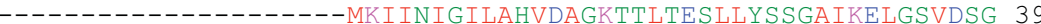
----------------MKI INLGILAHVDAGKTTLTESLIYTSGAIAEPGSVDKG 39

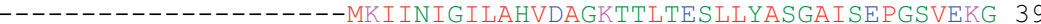
-------------------MKIINIGILAHVDAGKTTVTEGLLYKSGAINKIGRVDNA 39 MFKRVYVYKNSFWKYKKRFFSKKLVNIGILAHIDAGKTTISEDILYOSKEIKVKGNINDQ 60
\end{abstract}

TTRTDNTLLERQRGITIQTGITSFQWENTKVNIIDTPGHMDFLAEVYRSLSVLDGAILLI 99 TTKTDTMFLERQRGITIQTAITSFQRENVKVNIVDTPGHMDFLADVYRSLSVLDGAILII 99 TTRTDTMNLERQRGITIQTAVTSFQWEDVKVNI IDTPGHMDFLAEVYRSLSVLDGAVLLV 99 TTRTDTMFLERQRGITIQAAVTSFQWHRCKVNIVDTPGHMDFLAEVYRSLAVLDGAILVI 99 TTTTDSMELERDRGITIRASTVSFNYNDTKVNIIDTPGHMDFIAEVERTLKVLDGAILVI 99 NTQLDFLKOERERGITIKSAYSCEEWNKIKVNLIDTPGHIDFSNETFISLCVLDKCIIVI 120

SAKDGVQAQTRILFHALRKMGIPTIFFINKIDQNGIDLSTVYQDIKEKLSAEIVIKQKVE 159 SAKDGVQSQTRILFHALRKMNIPI IFFINKIDQNGINLPDVYQDIKDKLSDDIIIKQTVN 159 SAKDGIOAOTRI LFHALOTMK I PTIFFINK IDOEGIDLPMVYOEMKAKLSSEIIVKOKVG 159 SAKDGVQAQTRILFHALRKMNIPTVIFINKIDQAGVDLQSVVQSVRDKLSADIIIKQTVS 159 SAKEGIQVQTKVIFNTLVKLNIPTLIFVNKIDRKGVCLDEIYTQIQEKLTSNLAIMQSVK 159 DSKEGVQIQTINIFRYIKEN-LPIYFFLNKMDINHIDIDSNFLSIKNRLTKKGLLITYPI 179

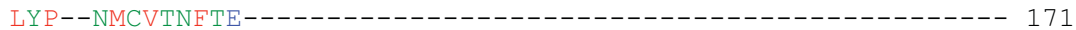

LNL--KPYVIDYTE----------------------------------------- 171

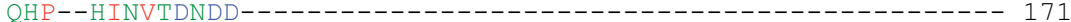

LSP--EIVLEENTD------------------------------------------- 171

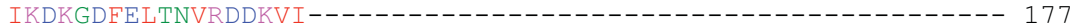

YENKKLKYILDIPSMVLYSYPHINYGQTFMYNSYFLKTLLEGSLYEKKLYSHFAMINPKP 239

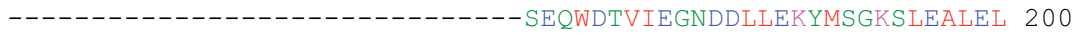

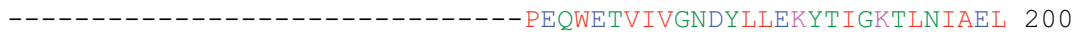

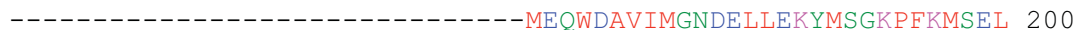

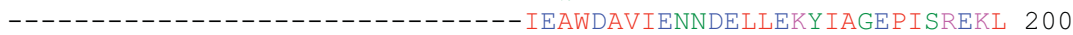
--_-_-_-_-_-_-_-_-_-_-_-_S I IEKLIDINDYLAEKY INGDVIAEKEY 207 DNLSKENKNKNNINIANVLNDDIIDYIIKKREEIIEILCDLDNTIEYKYLNNIPISYEHF 299

EQEESIRFHNCSLEPVYHGSAKNNIGIDNLIEVITN----------------------- 236

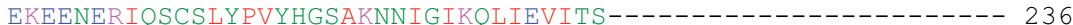
EQEENRRFQNGTLFPVYHGSAKNNLGIRQLIEVIAS---------------------- 236

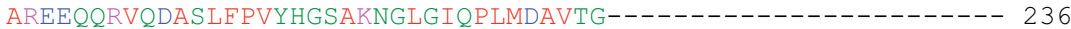
NDVFLDEINNCNLYPVFHGSALKNIGIDELIFAITK----------------------- 243 KNSLSLCIDKKYIFPIFSGSALHSYGVHILLDYITHNEKGMEKGMEKGMEKGMEKGMEKG 359

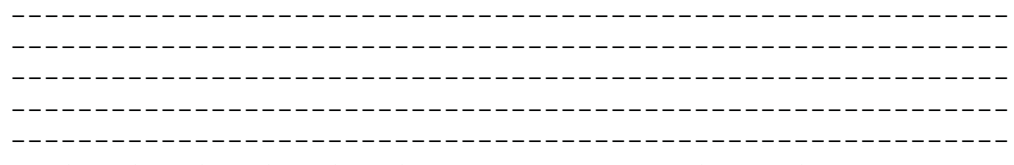

MEKGMEKGMEKGMEKGMEKGMEKGMENEMEMDMEKKMEKERQMNHNKQRTNNNTNETPFL 419

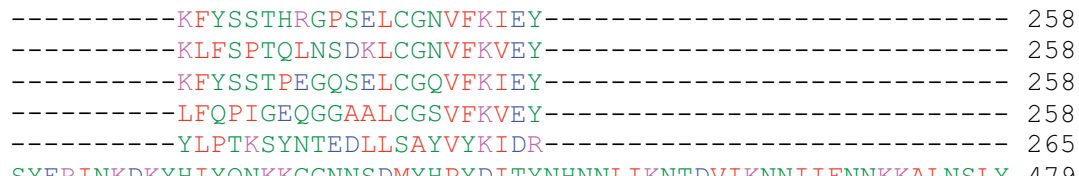

SYERTNKDKYHTYONKKCGNISDMYHDYDTTYNHNNT TKNTTDUTKNNTIFNNKKATNSTY 479

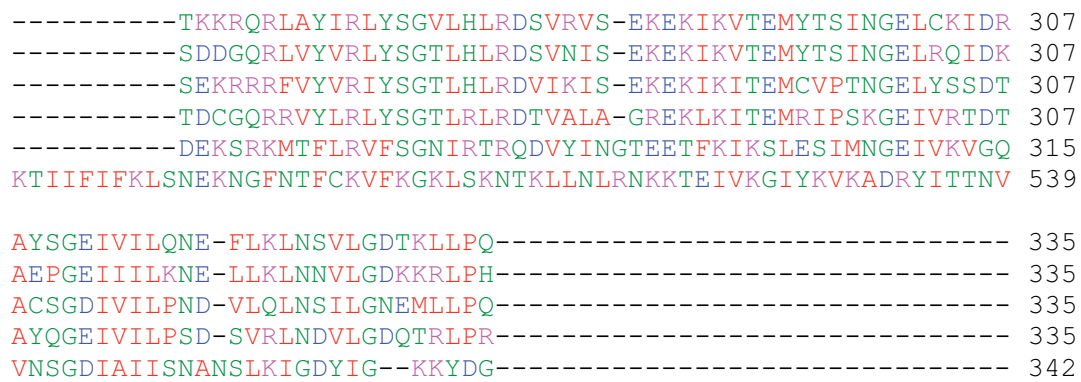

LDTNDIGMVRGFENIILCDIICEKDDEKPSHNNNDFFSEDQTTDHNKQNNEYSHSNHIEN 599

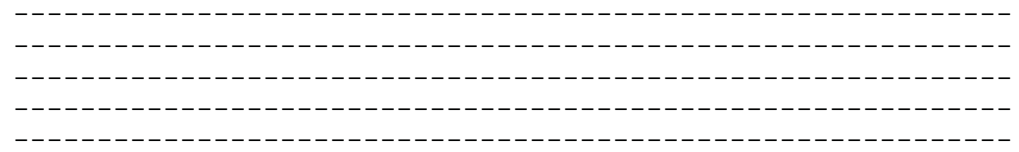


(Fig. 2) contd....

\begin{tabular}{|c|c|c|}
\hline AAN36428 & TLNELYHKSHKEDEKNHALHEGTYSQNNLF I GHNDLPPLSNIYKLLKDEI PNKQWLYFLK & 659 \\
\hline CAA52967 & --RKKIENPHPLLRTTVEPSKPEQREMLLDALLEISDSDPLLRYYVDSTTHEIILSFLGK & 393 \\
\hline Q48791 & --REILENPLPMLQTTIEPCKSVQREKLLDALFEISDSDPLLQYYVDTVTHEIVLSFLGE & 393 \\
\hline CAA69103 & --RKFIENPLPMLQTTIAVKKSEQREILLGALTEISDGDPLLKYYVDTTTHEIILSFLGN & 393 \\
\hline CAD20560 & --KRWREDPLPMLRTTIAPKTAAQRERLIDALTQLADTDPLLRCEVDSITHEIILSFLGR & 393 \\
\hline AAF01499 & --ILDIKIAQPALRAS IKPCDLSKRSKLIEALFELTEEDPFLDCE INGDTGEI ILRLFGN & 400 \\
\hline AAN36428 & SYKKRISKNI IVCTCAIEPKEYKKEKDLKNILKQICLEDNSILIFTD-KNNKLVIGSIGI & 718 \\
\hline CAA52967 & VQMEVISALIQEKYHVEIELKEPTVIYMERPLKNAEYTIHIEVPPNPFWASIGLSVSP-- & 451 \\
\hline Q48791 & VQMEVTCTLIQEKYHIEIETRKPTVIYMERPLKKSEFTIDIEVPPNPFWASIGLSVTP-- & 451 \\
\hline CAA69103 & VQMEVICAILEEKYHVEAEIKEPTVIYMERPLRKAEYTIHIEVPPNPFWASVGLSIEP-- & 451 \\
\hline CAD2 0560 & VQLEVVSALLSEKYKLETVVKEPSVIYMERPLKAASHTIHIEVPPNPFWASIGLSVTP-- & 451 \\
\hline AAF01499 & IQMEVIESLLKSRYKIDARFGELKTIYKERPKRNSKAVIHIEVPPNPYWASIGLSIEP-- & 458 \\
\hline AAN36428 & LNIEVI IDK IKNDYNIDIKTSPVEI IQKEY IQGYYENS IKKEMKVGSY ISTI I LGFVIKE & 778 \\
\hline CAA52967 & ----- & \\
\hline Q48791 & 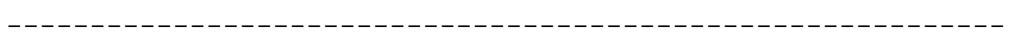 & \\
\hline CAA69103 & 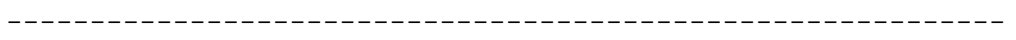 & \\
\hline CAD20560 & 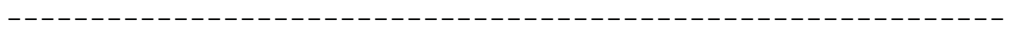 & \\
\hline AAF01499 & 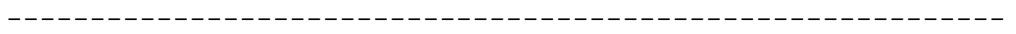 & \\
\hline AAN36428 & KDEFIDISSYVQNVLKHEKISHFLSSEEG I KNN I SNNKYNNNNKYNNNNKYNNNNKLNIS & 838 \\
\hline CAA52967 & 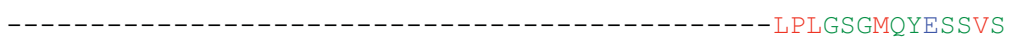 & 465 \\
\hline Q48791 & 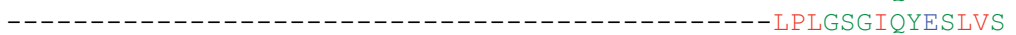 & 465 \\
\hline CAA69103 & 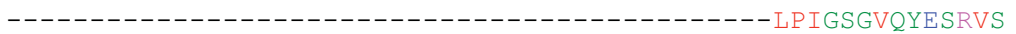 & 465 \\
\hline CAD2 0560 & 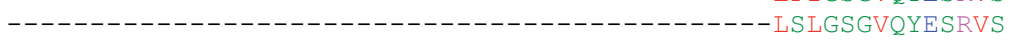 & 465 \\
\hline AAF01499 & --------------------------------------LPI GS GLIYKTEVS & 472 \\
\hline AAN36428 & DNLDKDNNLLLYDDIREEDNKKMY ISTTNDDRQNYDEHHN IN I LDNME IKESTEKDRKKN & 898 \\
\hline 967 & LGYLNQSFQNAVMEGIRYGCEQGLYGWNVTDCKICFKYGLYYSPVSTPADFR & 517 \\
\hline Q 48791 & LGYLNQSFQNAVMEGIRYGCEQGLYGWKLTDCKICFKYGLYYSPVSTPADFR-------- & 517 \\
\hline CAA69103 & LGYLNQSFQNAVMEGVLYGCEQGLYGWKVTDCKICFEYGLYYSPVSTPADFR------- & 517 \\
\hline CAD20560 & LGYLNQSFQNAVRDGIRYGLEQGLFGWNVTDCKICFEYGLYYSPVSTPADFR------- & 517 \\
\hline AAF01499 & YGYLNNSFQNAVKDAVEKACKEGLYGWEVTDLKVTFDYGLYYSPVSTPSDFR-------- & 524 \\
\hline AAN3 6428 & YVYNNLKLGNSKSMYDTKGVKNWVHKYNDDHDKIYLEDNI KDHPHKQS I DDEPELLCDND & 958 \\
\hline CAA52967 & ----MLAPIVLEQVLKKAGTELLEPYLS & 541 \\
\hline Q48791 & - & 541 \\
\hline CAA69103 & 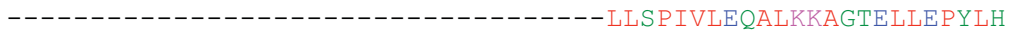 & 541 \\
\hline CAD2 0560 & 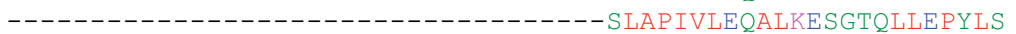 & 541 \\
\hline AAF01499 & 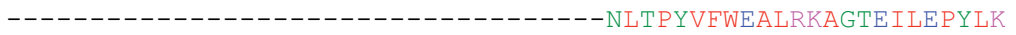 & 548 \\
\hline AAN36428 & DNDDNDNDDDNDDVDEYLLNFNYDTLFENSVTVHKDVLLYIDELKKMNKKKKNVYDNILN & 1018 \\
\hline CAA52967 & 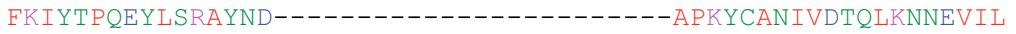 & 577 \\
\hline Q48791 & 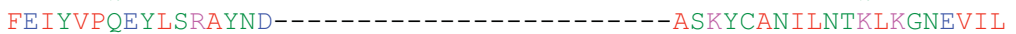 & 577 \\
\hline CAA69103 & 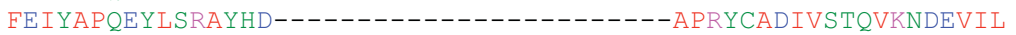 & 577 \\
\hline CAD2 0560 & FILYAPQEYLSRAYHD---------------------APKYCATIETAQVKKDEVVF & 577 \\
\hline AAF01499 & 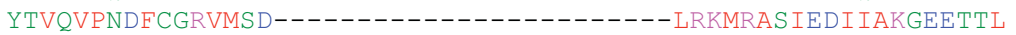 & 584 \\
\hline AAN36428 & SCI I S LKNCLSNGYHTNGNI INTEI I IKNLKIFDSSTTAVAKYACNHLYYEMIKKANIQI & 1078 \\
\hline CAA52967 & SGEIPARCIQEYRS-------DLTFFTNGRSVCLTELKG--YHVTTGEPVCQPR---RPN & 625 \\
\hline Q48791 & IGEIPARCIQEYRN-------SLTFFTNGRSVCLTELKG--YQVTNIKSAFQPR---RPN & 625 \\
\hline CAA69103 & KGEIPARCIQEYRN-------DLTYFTNGQGVCLTELKG--YQPAIGKFICQPR---RPN & 625 \\
\hline CAD20560 & TGEIPARCIQAYRT------DLAFYTNGRSVCLTELKG--YQAAVGQPVIQPR---RPN & 625 \\
\hline AAF01499 & SGKIPVDTSKSYQS------ELLSYSNGKGIFITEPYG--YDIYNDKPIINDIGNDNND & 635 \\
\hline AAN36428 & VNPLSLILIQTDEAYTGI IVKDLIQYRNGTI IQIMKNKESDFKLMKIYAI I PVKFTHNYS & 1138 \\
\hline CAA52967 & SRIDKVRYMFNKIT--------- 639 & \\
\hline Q48791 & NRIDKVRHMFNKINLH------- 641 & \\
\hline CAA69103 & SRIDKVRHMFHKLA--------- 639 & \\
\hline CAD20560 & SRLDKVRHMFQKVM--------- 639 & \\
\hline AAF01499 & SNKEGLRYLFQKQDEN------- 651 & \\
\hline AAN36428 & SILRSISSGHANFLMTFCGYKKC 1161 & \\
\hline
\end{tabular}

The NCBI accession numbers AAF01499, Q48791, CAA52967, CAA69103, CAD20560 and AAN36428 correspond respectively to tetT (Streptococcus pyogenes), tetS (Listeria monocytogenes), tetM (Neisseria meningitidis), tetO (Streptococcus pneumoniae), tetW (Butyrivibrio fibrisolvens) and tetQ family GTPase putative (Plasmodium falciparum 3D7). Residues are coloured according to their physical and chemical properties: hydrophobic $=$ red, basic $=$ pink, acidic $=$ blue, hydrophilic $=$ green .

Fig. (2). CLUSTALW alignment of five bacterial ribosomal protection protein sequences and TetQ GTPase family putative protein of Plasmodium falciparum. 


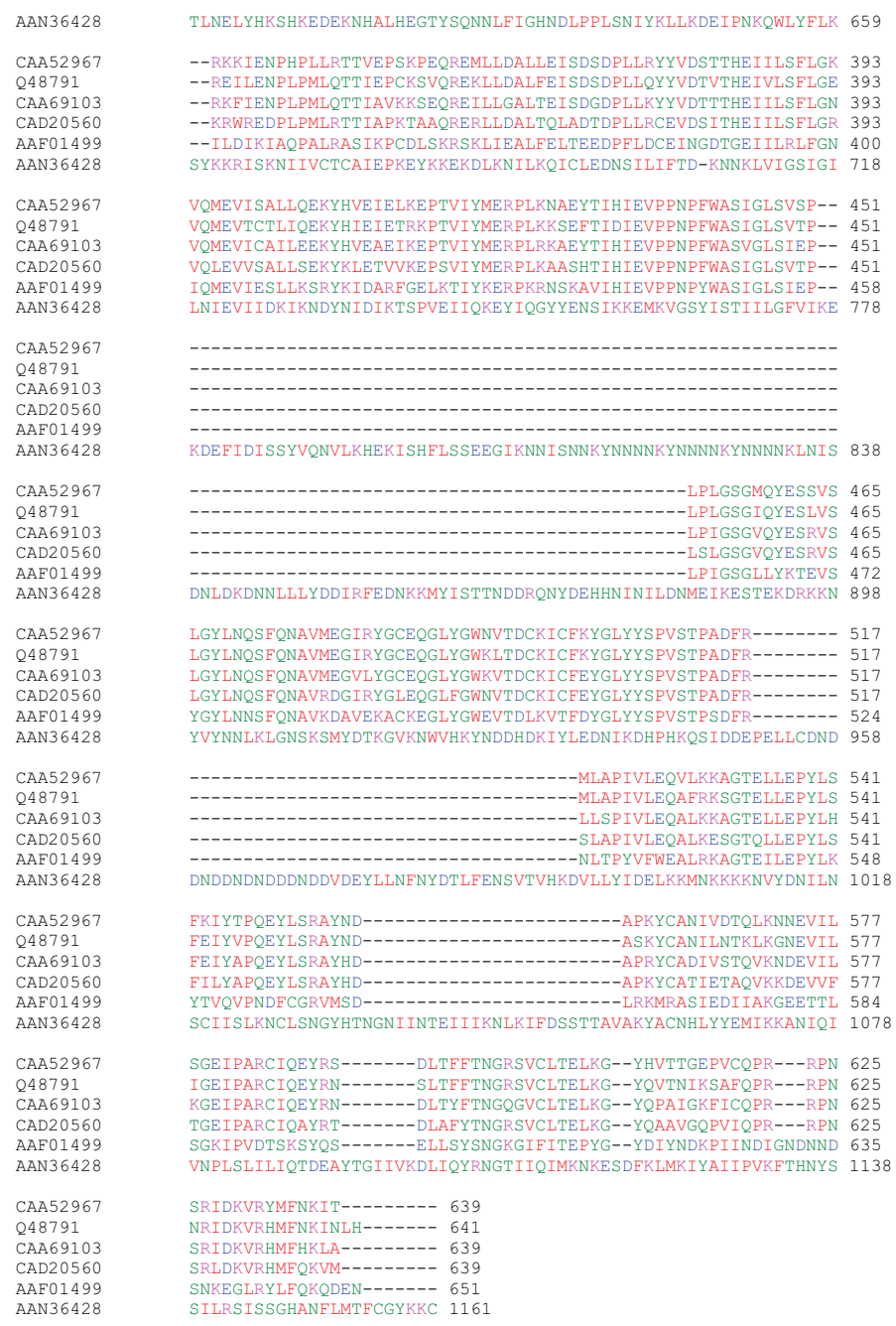

The three-dimensional structure of EFG of Thermus thermophilus was obtained from X-ray crystallography data and is available in the protein database (accession number $1 \mathrm{ktv}$ ). EFG is dimeric and has two chains A and B with four functional domains: EFTu is coloured in red, EFTu-D2 in blue, EFG-IV in orange and EFG-C in magenta. This picture was realized with the software PyMOL version 0.98 .

\section{Fig. (3). Three-dimensional structure of Elongation Factor G of Thermus thermophilus.}

were never documented with respect to a resistance to doxycycline.

\section{DOXYCYCLINE TOLERANCE}

Doxycycline is counter-indicated in cases of allergies to cyclines, in pregnant women from the second quarter of pregnancy due to a risk of anomalies of the dental bud (no teratogenic effect was observed in animal models and some pregnant women treated with cyclines) and in children younger than eight years due to the risk of dyschromy and hypoplasia of dental enamel. Association with retinoid is also counter-indicated, as it can generate an intracranial hypertension. Association with anticoagulants (antivitamine K) is to be avoided because it may induce hemorrhagic accidents.

The side effects of cyclines are known in the treatment of the bacterial infections. Their tolerance is good in the long treatment course (several months up to five years) of acne at a dose of $250 \mathrm{mg}$ of oxytetracycline twice a day, or $100 \mathrm{mg}$ of minocycline twice a day $[108,109]$, and do not require any particular biological follow-up [110]. For malaria che- moprophylaxis, this may imply use of doxycycline for several months at low dose (100 $\mathrm{mg}$ per day). The following principal side effects reported in the literature are in order of frequency:

- digestive disorders (in 2.5 to $20 \%$ of the cases according to studies'). Rare esophagitis and oesophageal ulcerations $[111,112]$ are practically no longer reported since the introduction of the monohydrate formula is less ulcerogenic than the old monohyclate formula [113];

- sleep disorders (in 1.3 to $14 \%$ of the cases) and headaches (approximately 10\%) are reported [97, 98, 114];

- cutaneous signs (in 0.6 to $8 \%$ of the cases according to authors') with urticarias, photosensitizations (the photo toxicity of doxycycline is a function of the dose, to 200 $\mathrm{mg} 50 \%$ of Caucasian subjects are photosensitized [115]), exfoliation, cutaneous rash [116], and sometimes photo-onycholysis [117];

- vaginal candidoses may occur in approximately $1 \%$ of the women [118]; 


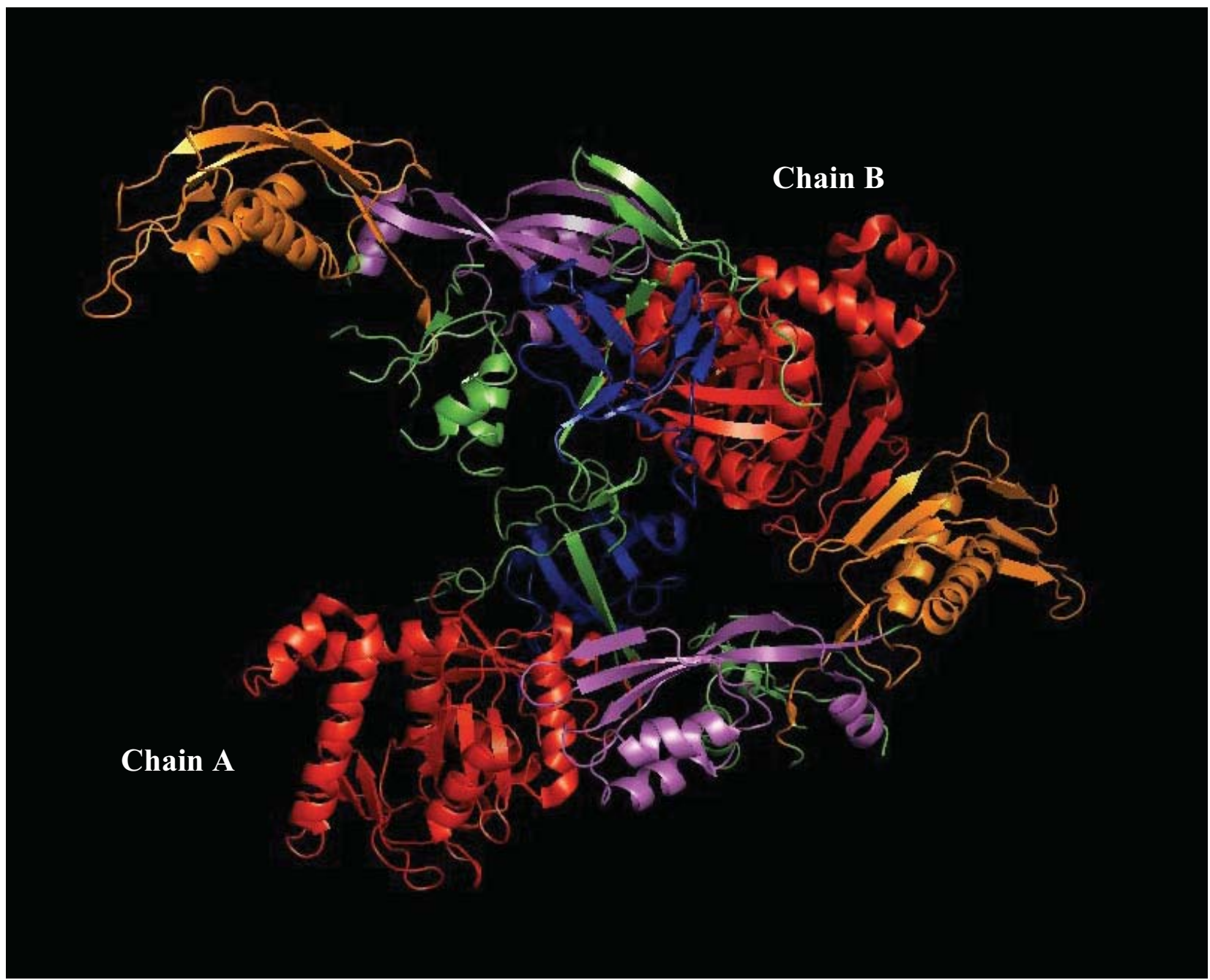

The three-dimensional structure of TetW of Butyrivibrio fibrisolvens (NCBI accession number CAD20560) was obtained from the amino-acid sequence using the prediction three-dimensional structure software 3D-JIGSAW version 2.0. TetW is monomeric with four functional domains similar to EFG: EFTu is coloured in red, EFTu-D2 in blue, EFG-IV in orange and EFG-C in magenta. This picture was realized with the software PyMOL version 0.98.

Fig. (4). Three-dimensional structure of TetW protein of Butyrivibrio fibrisolvens.

- two cases of intracranial hypertension were listed among patients without cardiovascular or neurological risk factors with doxycycline during a malaria chemoprophylaxis [119].

Thus, doxycycline tolerance is effective and equivalent to the majority of the other antimalarial drugs used in malaria prevention.

The use of an antibiotic for several months in malaria chemoprophylaxis may render the bacteria resistant to the cyclines (the molecular epidemiology and the bacterial mechanisms of resistance to the cyclines were largely described by Chopra in 2001 [14]). In 1988, the first publication of gastroenteritis to Campylobacter jejuni resistant to tetracyclines in American soldiers serving in Thailand [120]. A later study undertaken by the same team has shown that doxycycline in malaria chemoprophylaxis exposes fewer to the selection of bacteria resistance than to the acquisition of bacteria already resistant to the cyclines that were largely widespread in this country for a long time [121]. Only one other study was published regarding the risk of emergence of bacterial resistances to the cyclines [122] associated with their use in malaria chemoprophylaxis.

\section{OUTLINES}

The emergence and the rapid extension of the resistance of $P$. falciparum to principal antimalarial drugs available necessitate the search for new molecules. In the meantime, doxycycline constitutes an excellent molecule for the treatment of uncomplicated malaria in zones of multi-resistances but only in association with fast schizontocide like quinine or artesunate. Doxycycline's good tolerance and its efficacy were proven. Doxycycline is currently the only antimalarial drug for which no resistance of $P$. falciparum was described so doxycycline use should be expanded in particular in the prevention to $P$. falciparum malaria except in pregnant women and in children less than eight years old. The major limitation would probably be the poor compliance because of the short half life and the weak activity on hepatic stages which imposes a daily dose of $100 \mathrm{mg}$ and its continuation during four weeks after the return from endemic areas. A better comprehension of the mechanisms of action of doxy- 


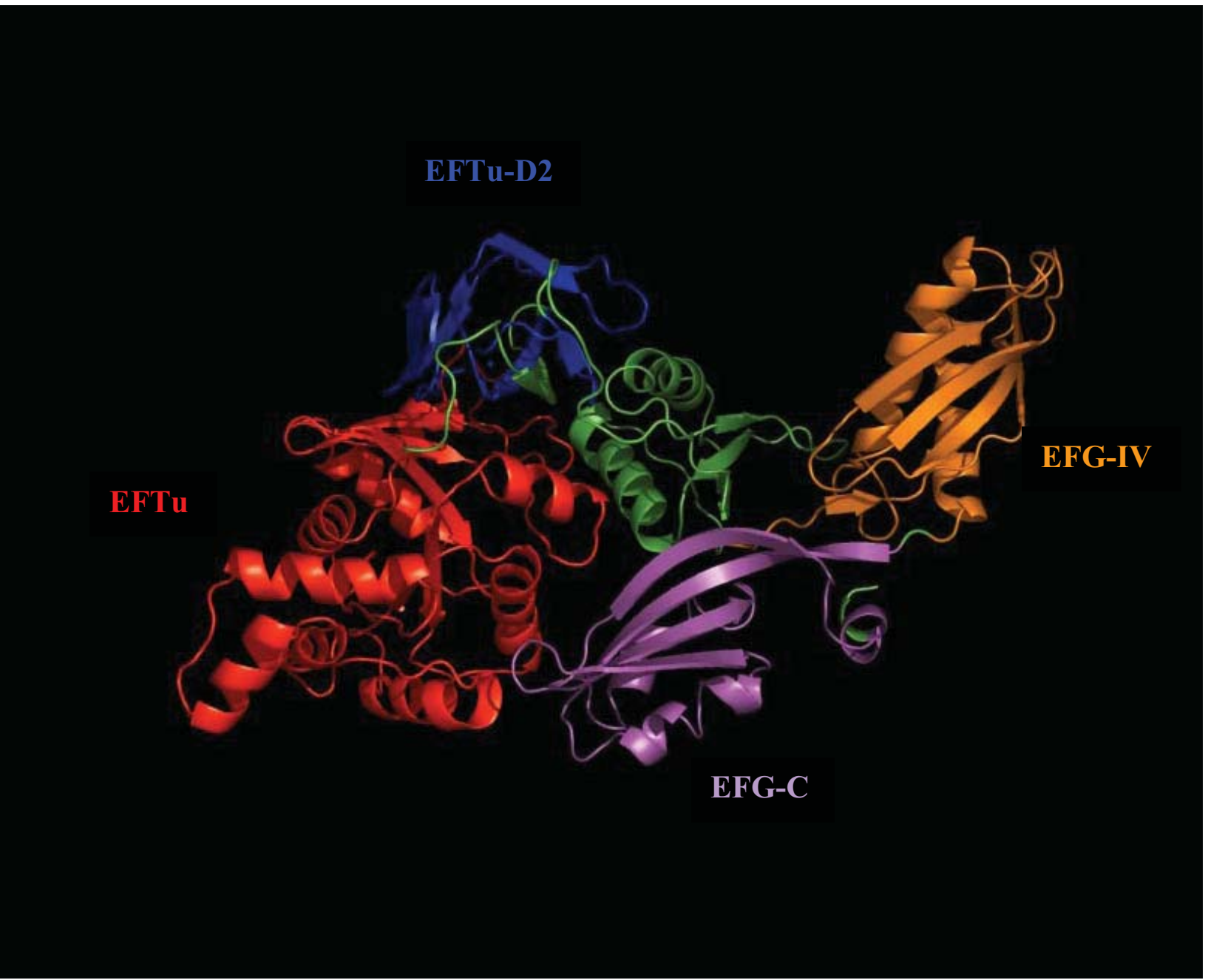

The three-dimensional structure of TetQ GTPase putative of Plasmodium falciparum (NCBI accession number PFL1710c) was obtained from the amino-acid sequence using the prediction three-dimensional structure software 3D-JIGSAW version 2.0. This protein possesses two functional domains: EFTu is coloured in red and EFG-C in magenta. Amino-acids coloured in blue are the same of TetW. EFG-C seems to contain the two domains EFG-IV and EFG-C of TetW. The EFTu-D2 domain of TetW might exist in TetQ as the similarities are higher in this region between the two proteins. This picture was realized with the software PyMOL version 0.98

\section{Fig. (5). Three-dimensional structure of TetQ GTPase family protein of Plasmodium falciparum 3D7.}

cycline on $P$. falciparum and the identification of its molecular targets would allow the design of more effective $\left(\mathrm{IC}_{50}\right.$ around $1 \mathrm{nM}$ ) and stable structural analogues.

\section{REFERENCES}

[1] Snow RW, Guerra CA, Noor AM, Myint HY, Hay SI. The global distribution of clinical episodes of Plasmodium falciparum malaria. Nature 2005; 434: 214-7.

[2] Trape JF, Pison G, Spiegel A, Enel C, Rogier C. Combating malaria in Africa. Trends Parasitol 2002; 18: 224-30.

[3] Rogier C, Orlandi-Pradines E, Fusai T, Pradines B, Briolant S, Almeras L. Malaria vaccines: Prospects and reality. Med Mal Infect 2006; 36: 414-22.

[4] Pradines B, Vial H, Olliaro P. Malaria prophylaxis and treatment: Problems, recent developments and perspectives. Med Trop 2003; 63: 79-98.

[5] Wellems TE, Plowe CV. Chloroquine-resistant malaria. J Infect Dis 2001; 184: 770-6.

[6] Le Bras J, Musset L, Clain J. Antimalarial drug resistance. Med Mal Infect 2006; 36: 401-5.

[7] Das B, Jena RK, Swain KP, Parida P. Emerging resistance of Plasmodium falciparum to artemisinine and related compounds. J Assoc Physicians India 2000; 48: 443-4.
[8] Pradines B, Rogier C, Fusai T, Tall A, Trape JF, Doury JC. In vitro activity of artemether against African isolates (Senegal) of Plasmodium falciparum in comparison with standard antimalarial drugs. Am J Trop Med Hyg 1998; 58: 354-7.

[9] Pradines B, Mabika MM, Parzy D, et al. In vitro susceptibility of Gabonese wild isolates of Plasmodium falciparum to artemether, and comparison with chloroquine, quinine, halofantrine and amodiaquine. Parasitology 1998; 117(Pt 6): 541-5.

[10] Jambou R, Legrand E, Niang M, et al. Resistance of Plasmodium falciparum field isolates to in-vitro artemether and point mutations of the SERCA-type PfATPase6. Lancet 2005; 366: 1960-3.

[11] Musset L, Bouchaud O, Matheron S, Massias L, Le Bras J. Clinical atovaquone-proguanil resistance of Plasmodium falciparum associated with cytochrome b codon 268 mutations. Microbes Infect 2006; 8: 2599-604.

[12] Gingras BA, Jensen JB. Activity of azithromycin (CP-62,993) and erythromycin against chloroquine-sensitive and chloroquine-resistant strains of Plasmodium falciparum in vitro. Am J Trop Med Hyg 1992; 47: 378-82.

[13] Kuschner RA, Heppner DG, Andersen SL, et al. Azithromycin prophylaxis against a chloroquine-resistant strain of Plasmodium falciparum. Lancet 1994; 343: 1396-7. 
[14] Chopra I, Roberts M. Tetracycline antibiotics: Mode of action, applications, molecular biology, and epidemiology of bacterial resistance. Microbiol Mol Biol Rev 2001; 65: 232-60.

[15] Debrah AY, Mand S, Specht S, et al. Doxycycline reduces plasma VEGF-C/sVEGFR-3 and improves pathology in lymphatic filariasis. PLoS Pathog 2006; 2(9): e92.

[16] Imboden CA Jr, Cooper WC, Coatney GR, Jeffery GM. Studies in human malaria. XXIX. Trials of aureomycin, chloramphenicol, penicillin, and dihydrostreptomycin against the Chesson strain of Plasmodium vivax. J Natl Malar Soc 1950; 9: 377-80.

[17] Ruiz Sanchez F, Casillas J, Paredes M, Velazquez J, Riebeling QB. Terramycin in malaria therapy. Pan Am Med Women J 1952; 59: 10-5.

[18] Grande EN, Sanchez AR, Sanchez FR. The treatment of malaria with tetracycline. Antibiotic Med Clin Ther 1956; 3: 193-6.

[19] Clyde DF, Miller RM, DuPont HL, Hornick RB. Antimalarial effects of tetracyclines in man. J Trop Med Hyg 1971; 74: 238-42.

[20] Willerson D, Rieckmann KH, Carson PE, Frischer H. Effects of minocycline against chloroquine-resistant falciparum malaria. Am J Trop Med Hyg 1972; 21: 857-62.

[21] Fabre J, Milek E, Kalfopoulos P, Merier G. Tetracycline kinetics in man. Digestive absorption and serum concentration. Schweiz Med Wochenschr 1971; 101: 593-8.

[22] Saivin S, Houin G. Clinical pharmacokinetics of doxycycline and minocycline. Clin Pharmacokinet 1988; 15: 355-66.

[23] Cunha BA, Sibley CM, Ristuccia AM. Doxycycline. Ther Drug Monit 1982; 4: 115-35.

[24] Welling PG, Koch PA, Lau CC, Craig WA. Bioavailability of tetracycline and doxycycline in fasted and nonfasted subjects. Antimicrob Agents Chemother 1977; 11: 462-9.

[25] Maibach H. Second-generation tetracyclines, a dermatologic overview: Clinical uses and pharmacology. Cutis 1991; 48: 411-7.

[26] Geary TG, Divo AA, Jensen JB. Uptake of antibiotics by Plasmodium falciparum in culture. Am J Trop Med Hyg 1988; 38: 466-9.

[27] Newton PN, Chaulet JF, Brockman A, et al. Pharmacokinetics of oral doxycycline during combination treatment of severe falciparum malaria. Antimicrob Agents Chemother 2005; 49: 1622-5.

[28] Pioletti M, Schlunzen F, Harms J, et al. Crystal structures of complexes of the small ribosomal subunit with tetracycline, edeine and IF3. EMBO J 2001; 20: 1829-39.

[29] Olson MW, Ruzin A, Feyfant E, Rush TS, 3rd, O'Connell J, Bradford PA. Functional, biophysical, and structural bases for antibacterial activity of tigecycline. Antimicrob Agents Chemother 2006; 50: 2156-66

[30] Oehler R, Polacek N, Steiner G, Barta A. Interaction of tetracycline with RNA: Photoincorporation into ribosomal RNA of Escherichia coli. Nucleic Acids Res 1997; 25: 1219-24.

[31] Anokhina MM, Barta A, Nierhaus KH, Spiridonova VA, Kopylov AM. Mapping of the second tetracycline binding site on the ribosomal small subunit of E.coli. Nucleic Acids Res 2004; 32: 2594-7.

[32] Sherman IW. The ribosomes of the simian malaria Plasmodium knowlesi--II. A cell-free protein synthesizing system. Comp Biochem Physiol B 1976; 53: 447-50.

[33] Divo AA, Geary TG, Jensen JB. Oxygen- and time-dependent effects of antibiotics and selected mitochondrial inhibitors on Plasmodium falciparum in culture. Antimicrob Agents Chemother 1985; 27: 21-7.

[34] Krungkrai J. The multiple roles of the mitochondrion of the malarial parasite. Parasitology 2004; 129: 511-24.

[35] Blum JJ, Yayon A, Friedman S, Ginsburg H. Effects of mitochondrial protein synthesis inhibitors on the incorporation of isoleucine into Plasmodium falciparum in vitro. J Protozool 1984; 31: 475-9.

[36] Kiatfuengfoo R, Suthiphongchai T, Prapunwattana P, Yuthavong Y. Mitochondria as the site of action of tetracycline on Plasmodium falciparum. Mol Biochem Parasitol 1989; 34: 109-15.

[37] Budimulja AS, Syafruddin, Tapchaisri P, Wilairat P, Marzuki S. The sensitivity of Plasmodium protein synthesis to prokaryotic ribosomal inhibitors. Mol Biochem Parasitol 1997; 84: 137-41.

[38] Prapunwattana P, Sullivan WJ, Yuthavong Y. Depression of Plasmodium falciparum dihydroorotate dehydrogenase activity in in vitro culture by tetracycline. Mol Biochem Parasitol 1988; 27: 11924.

[39] Yeo AE, Rieckmann KH, Christopherson RI. Indirect inhibition by antibiotics of nucleotide and deoxynucleotide biosynthesis in Plasmodium falciparum. Southeast Asian J Trop Med Public Health 1998; 29: 24-6.
[40] Lin Q, Katakura K, Suzuki M. Inhibition of mitochondrial and plastid activity of Plasmodium falciparum by minocycline. FEBS Lett 2002; 515: 71-4.

[41] Dahl EL, Shock JL, Shenai BR, Gut J, DeRisi JL, Rosenthal PJ. Tetracyclines specifically target the apicoplast of the malaria parasite Plasmodium falciparum. Antimicrob Agents Chemother 2006; 50: 3124-31.

[42] Goodman CD, Su V, McFadden GI. The effects of anti-bacterials on the malaria parasite Plasmodium falciparum. Mol Biochem Parasitol 2007; 152: 181-91.

[43] Dahl EL, Rosenthal PJ. Multiple antibiotics exert delayed effects against the Plasmodium falciparum apicoplast. Antimicrob Agents Chemother 2007; 51: 3485-90.

[44] Fichera ME, Roos DS. A plastid organelle as a drug target in apicomplexan parasites. Nature 1997; 390: 407-9.

[45] Garnham PC, Warren M, Killick-Kendrick R. The action of 'terramycin' on the primary exoerythrocytic development of Plasmodium vivax and Plasmodium cynomolgi ceylonensis. J Trop Med Hyg 1971; 74: 32-5.

[46] Kumar A, Dutta GP. Tissue schizontocidal activity of minocycline against a relapsing malaria parasite Plasmodium cynomolgi B. Indian J Med Res 1987; 85: 519-21.

[47] Kumar A, Dutta GP. Antimalarial activity of demeclocycline against Plasmodium cynomolgi bastianellii in rhesus monkeys. Ann Trop Med Parasitol 1989; 83: 199-206.

[48] Marussig M, Motard A, Renia L, et al. Activity of doxycycline against preerythrocytic malaria. J Infect Dis 1993; 168: 1603-4.

[49] Shmuklarsky MJ, Boudreau EF, Pang LW, et al. Failure of doxycycline as a causal prophylactic agent against Plasmodium falciparum malaria in healthy nonimmune volunteers. Ann Intern Med 1994; 120: 294-9.

[50] Orlandi-Pradines E, Penhoat K, Durand C, et al. Antibody responses to several malaria pre-erythrocytic antigens as a marker of malaria exposure among travelers. Am J Trop Med Hyg 2006; 74 : 979-85.

[51] Geary TG, Divo AA, Jensen JB. Stage specific actions of antimalarial drugs on Plasmodium falciparum in culture. Am J Trop Med Hyg 1989; 40: 240-4.

[52] Basco LK, Le Bras J. Activity in vitro of doxycycline against multidrug-resistant Plasmodium falciparum. Trans R Soc Trop Med Hyg 1993; 87: 469-70.

[53] Pradines B, Spiegel A, Rogier C, et al. Antibiotics for prophylaxis of Plasmodium falciparum infections: in vitro activity of doxycycline against Senegalese isolates. Am J Trop Med Hyg 2000; 62: 82-5.

[54] Gershon PD, Howells RE. Combination of the antibiotics erythromycin and tetracycline with three standard antimalarials against Plasmodium falciparum in vitro. Ann Trop Med Parasitol 1984; 78: $1-11$.

[55] Canfield CJ, Pudney M, Gutteridge WE. Interactions of atovaquone with other antimalarial drugs against Plasmodium falciparum in vitro. Exp Parasitol 1995; 80: 373-81.

[56] Yeo AE, Edstein MD, Shanks GD, Rieckmann KH. Potentiation of the antimalarial activity of atovaquone by doxycycline against Plasmodium falciparum in vitro. Parasitol Res 1997; 83: 489-91.

[57] Ye Z, Van Dyke K. Interaction of artemisinin and tetracycline or erythromycin against Plasmodium falciparum in vitro. Parasite 1994; 1: 211-8.

[58] Sponer U, Prajakwong S, Wiedermann G, Kollaritsch H, Wernsdorfer G, Wernsdorfer WH. Pharmacodynamic interaction of doxycycline and artemisinin in Plasmodium falciparum. Antimicrob Agents Chemother 2002; 46: 262-4.

[59] Fivelman QL, Walden JC, Smith PJ, Folb PI, Barnes KI. The effect of artesunate combined with standard antimalarials against chloroquine-sensitive and chloroquine-resistant strains of Plasmodium falciparum in vitro. Trans R Soc Trop Med Hyg 1999; 93: 429-32.

[60] Batty KT, Law AS, Stirling V, Moore BR. Pharmacodynamics of doxycycline in a murine malaria model. Antimicrob Agents Chemother 2007; 51: 4477-9.

[61] Rieckmann KH, Powell RD, McNamara JV, et al. Effects of tetracycline against chloroquine-resistant and chloroquine-sensitive Plasmodium falciparum. Am J Trop Med Hyg 1971; 20: 811-5.

[62] Laing AB. The effect of tetracycline on Plasmodium falciparum in the Gambia. Trans R Soc Trop Med Hyg 1972; 66: 956-7. 
[63] Ponnampalam JT. Doxycycline in the treatment of falciparum malaria among aborigine children in West Malaysia. Trans R Soc Trop Med Hyg 1981; 75: 372-7.

[64] Pukrittayakamee S, Clemens R, Chantra A, et al. Therapeutic responses to antibacterial drugs in vivax malaria. Trans R Soc Trop Med Hyg 2001; 95: 524-8.

[65] Colwell EJ, Hickman RL, Kosakal S. Tetracycline treatment of chloroquine-resistant falciparum malaria in Thailand. JAMA 1972; 220: 684-6.

[66] Chin W, Intraprasert R. The evaluation of quinine alone or in combination with tetracycline and pyrimethamine against falciparum malaria in Thailand. Southeast Asian J Trop Med Public Health 1973; 4: 245-9.

[67] Colwell EJ, Hickman RL, Kosakal S. Quinine-tetracycline and quinine-bactrim treatment of acute falciparum malaria in Thailand. Ann Trop Med Parasitol 1973; 67: 125-32.

[68] Noeypatimanond S, Malikul S, Benjapong W, Duriyananda D, Ungkasrithongkul M. Treatment of Plasmodium falciparum malaria with a combination of amodiaquine and tetracycline in Central Thailand. Trans R Soc Trop Med Hyg 1983; 77: 338-40.

[69] Giboda M, Denis MB. Response of Kampuchean strains of Plasmodium falciparum to antimalarials: In-vivo assessment of quinine and quinine plus tetracycline; multiple drug resistance in vitro. J Trop Med Hyg 1988; 91: 205-11.

[70] Looareesuwan S, Vanijanonta S, Viravan C, et al. Randomised trial of mefloquine-tetracycline and quinine-tetracycline for acute uncomplicated falciparum malaria. Acta Trop 1994; 57: 47-53.

[71] Looareesuwan S, Viravan C, Vanijanonta S, et al. Randomized trial of mefloquine-doxycycline, and artesunate-doxycycline for treatment of acute uncomplicated falciparum malaria. Am J Trop Med Hyg 1994; 50: 784-9.

[72] Na-Bangchang K, Kanda T, Tipawangso P, et al. Activity of artemether-azithromycin versus artemether-doxycycline in the treatment of multiple drug resistant falciparum malaria. Southeast Asian J Trop Med Public Health 1996; 27: 522-5.

[73] Looareesuwan S, Viravan C, Webster HK, Kyle DE, Hutchinson DB, Canfield CJ. Clinical studies of atovaquone, alone or in combination with other antimalarial drugs, for treatment of acute uncomplicated malaria in Thailand. Am J Trop Med Hyg 1996; 54: 62-6.

[74] Duarte EC, Fontes CJ, Gyorkos TW, Abrahamowicz M. Randomized controlled trial of artesunate plus tetracycline versus standard treatment (quinine plus tetracycline) for uncomplicated Plasmodium falciparum malaria in Brazil. Am J Trop Med Hyg 1996; 54 : 197-202.

[75] Bunnag D, Karbwang J, Na-Bangchang K, Thanavibul A, Chittamas S, Harinasuta T. Quinine-tetracycline for multidrug resistant falciparum malaria. Southeast Asian J Trop Med Public Health 1996; 27: 15-8.

[76] Pukrittayakamee S, Chantra A, Vanijanonta S, Clemens R, Looareesuwan S, White NJ. Therapeutic responses to quinine and clindamycin in multidrug-resistant falciparum malaria. Antimicrob Agents Chemother 2000; 44: 2395-8

[77] Taylor WR, Widjaja H, Richie TL, et al. Chloroquine/doxycycline combination versus chloroquine alone, and doxycycline alone for the treatment of Plasmodium falciparum and Plasmodium vivax malaria in northeastern Irian Jaya, Indonesia. Am J Trop Med Hyg 2001; 64: 223-8

[78] Pukrittayakamee S, Chotivanich K, Chantra A, Clemens R, Looareesuwan S, White NJ. Activities of artesunate and primaquine against asexual- and sexual-stage parasites in falciparum malaria. Antimicrob Agents Chemother 2004; 48: 1329-34.

[79] Alecrim MG, Lacerda MV, Mourao MP, et al. Successful treatment of Plasmodium falciparum malaria with a six-dose regimen of artemether-lumefantrine versus quinine-doxycycline in the Western Amazon region of Brazil. Am J Trop Med Hyg 2006; 74: 20-5.

[80] Metzger W, Mordmuller B, Graninger W, Bienzle U, Kremsner PG. High efficacy of short-term quinine-antibiotic combinations for treating adult malaria patients in an area in which malaria is hyperendemic. Antimicrob Agents Chemother 1995; 39: 245-6.

[81] Griffith KS, Lewis LS, Mali S, Parise ME. Treatment of malaria in the United States: A systematic review. JAMA 2007; 297: 2264-77.

[82] Watt G, Loesuttivibool L, Shanks GD, et al. Quinine with tetracycline for the treatment of drug-resistant falciparum malaria in Thailand. Am J Trop Med Hyg 1992; 47: 108-11.
[83] Pang LW, Limsomwong N, Boudreau EF, Singharaj P. Doxycycline prophylaxis for falciparum malaria. Lancet 1987; 1: 1161-4.

[84] Pang L, Limsomwong N, Singharaj P. Prophylactic treatment of vivax and falciparum malaria with low-dose doxycycline. J Infect Dis 1988; 158: 1124-7.

[85] Watanasook C, Singharaj P, Suriyamongkol V, et al. Malaria prophylaxis with doxycycline in soldiers deployed to the ThaiKampuchean border. Southeast Asian J Trop Med Public Health 1989; 20: 61-4.

[86] Shanks GD, Edstein MD, Suriyamongkol V, Timsaad S, Webster HK. Malaria chemoprophylaxis using proguanil/dapsone combinations on the Thai-Cambodian border. Am J Trop Med Hyg 1992; 46: 643-8.

[87] Weiss WR, Oloo AJ, Johnson A, Koech D, Hoffman SL. Daily primaquine is effective for prophylaxis against falciparum malaria in Kenya: Comparison with mefloquine, doxycycline, and chloroquine plus proguanil. J Infect Dis 1995; 171: 1569-75.

[88] Ohrt C, Richie TL, Widjaja H, et al. Mefloquine compared with doxycycline for the prophylaxis of malaria in Indonesian soldiers: A randomized, double-blind, placebo-controlled trial. Ann Intern Med 1997; 126: 963-72.

[89] Andersen SL, Oloo AJ, Gordon DM, et al. Successful doubleblinded, randomized, placebo-controlled field trial of azithromycin and doxycycline as prophylaxis for malaria in western Kenya. Clin Infect Dis 1998; 26: 146-50.

[90] Taylor WR, Richie TL, Fryauff DJ, et al. Malaria prophylaxis using azithromycin: a double-blind, placebo-controlled trial in Irian Jaya, Indonesia. Clin Infect Dis 1999; 28: 74-81.

[91] Rieckmann KH, Yeo AE, Davis DR, Hutton DC, Wheatley PF, Simpson R. Recent military experience with malaria chemoprophylaxis. Med J Aust 1993; 158: 446-9.

[92] Shanks GD, Roessler P, Edstein MD, Rieckmann KH. Doxycycline for malaria prophylaxis in Australian soldiers deployed to United Nations missions in Somalia and Cambodia. Mil Med 1995; 160 : 443-5.

[93] Shanks GD, Barnett A, Edstein MD, Rieckmann KH. Efficacy of doxycycline combined with primaquine for malaria prophylaxis. Med J Aust 1995; 162: 306-7, 309-10.

[94] Baudon D, Martet G, Pascal B, Bernard J, Keundjian A, Laroche R. Efficacy of daily antimalarial chemoprophylaxis in tropical Africa using either doxycycline or chloroquine-proguanil: A study conducted in 1996 in the French Army. Trans R Soc Trop Med Hyg 1999; 93: 302-3.

[95] Schwartz E, Regev-Yochay G. Primaquine as prophylaxis for malaria for nonimmune travelers: A comparison with mefloquine and doxycycline. Clin Infect Dis 1999; 29: 1502-6.

[96] Peragallo MS, Croft AM, Kitchener SJ. Malaria during a multinational military deployment: The comparative experience of the Italian, British and Australian Armed Forces in East Timor. Trans R Soc Trop Med Hyg 2002; 96: 481-2.

[97] Sonmez A, Harlak A, Kilic S, et al. The efficacy and tolerability of doxycycline and mefloquine in malaria prophylaxis of the ISAF troops in Afghanistan. J Infect 2005; 51: 253-8.

[98] Wallace MR, Sharp TW, Smoak B, et al. Malaria among United States troops in Somalia. Am J Med 1996; 100: 49-55.

[99] Paulsen IT, Brown MH, Skurray RA. Proton-dependent multidrug efflux systems. Microbiol Rev 1996; 60: 575-608.

[100] Blanco M, Kadlec K, Gutierrez Martin CB, de la Fuente AJ, Schwarz S, Navas J. Nucleotide sequence and transfer properties of two novel types of Actinobacillus pleuropneumoniae plasmids carrying the tetracycline resistance gene tet(H). J Antimicrob Chemother 2007; 60: 864-7.

[101] Yang W, Moore IF, Koteva KP, Bareich DC, Hughes DW, Wright GD. TetX is a flavin-dependent monooxygenase conferring resistance to tetracycline antibiotics. J Biol Chem 2004; 279: 52346-52.

[102] Nonaka L, Connell SR, Taylor DE. 16S rRNA mutations that confer tetracycline resistance in Helicobacter pylori decrease drug binding in Escherichia coli ribosomes. J Bacteriol 2005; 187: 370812 .

[103] Dantley KA, Dannelly HK, Burdett V. Binding interaction between Tet(M) and the ribosome: requirements for binding. J Bacteriol 1998; 180: 4089-92.

[104] Trieber CA, Burkhardt N, Nierhaus KH, Taylor DE. Ribosomal protection from tetracycline mediated by $\operatorname{Tet}(\mathrm{O})$ : $\operatorname{Tet}(\mathrm{O})$ interaction with ribosomes is GTP-dependent. Biol Chem 1998; 379: 847-55. 
[105] Kobayashi T, Nonaka L, Maruyama F, Suzuki S. Molecular evidence for the ancient origin of the ribosomal protection protein that mediates tetracycline resistance in bacteria. J Mol Evol 2007; 65: 228-35.

[106] Thompson JD, Higgins DG, Gibson TJ. CLUSTAL W: Improving the sensitivity of progressive multiple sequence alignment through sequence weighting, position-specific gap penalties and weight matrix choice. Nucleic Acids Res 1994; 22: 4673-80.

[107] Jacobs RL, Koontz LC. Plasmodium berghei: Development of resistance to clindamycin and minocycline in mice. Exp Parasitol 1976; 40: 116-23.

[108] Delaney TJ, Leppard BJ, MacDonald DM. Effects of long term treatment with tetracycline. Acta Derm Venereol 1974; 54: 487-9.

[109] Goulden V, Glass D, Cunliffe WJ. Safety of long-term high-dose minocycline in the treatment of acne. Br J Dermatol 1996; 134: 693-5.

[110] Driscoll MS, Rothe MJ, Abrahamian L, Grant-Kels JM. Long-term oral antibiotics for acne: is laboratory monitoring necessary? J Am Acad Dermatol 1993; 28: 595-02.

[111] Tzianetas I, Habal F, Keystone JS. Short report: Severe hiccups secondary to doxycycline-induced esophagitis during treatment of malaria. Am J Trop Med Hyg 1996; 54: $203-4$.

[112] Morris TJ, Davis TP. Doxycycline-induced esophageal ulceration in the US military service. Mil Med 2000; 165: 316-9.

[113] Carlborg B, Farmer J. Esophageal corrosion tests with doxycycline monohydrate tablets. Curr Ther Res 1983; 34: 110-5.
[114] Kitchener SJ, Nasveld PE, Gregory RM, Edstein MD. Mefloquine and doxycycline malaria prophylaxis in Australian soldiers in East Timor. Med J Aust 2005; 182: 168-71.

[115] Loesche C, Bocquet J, Bonnevalle A, Fertin V, Thomas P. Etude de la phototoxicité chez 30 sujets traités par la doxycycline à différentes doses. Nouv Dermatol 1994; 13: 383-8.

[116] Pages F, Boutin JP, Meynard JB, et al. Tolerability of doxycycline monohydrate salt vs chloroquine-proguanil in malaria chemoprophylaxis. Trop Med Int Health 2002; 7: 919-24.

[117] Rabar D, Combemale P, Peyron F. Doxycycline-induced photoonycholysis. J Travel Med 2004; 11: 386-7.

[118] Korhonen C, Peterson K, Bruder C, Jung P. Self-reported adverse events associated with antimalarial chemoprophylaxis in peace corps volunteers. Am J Prev Med 2007; 33: 194-9.

[119] Lochhead J, Elston JS. Doxycycline induced intracranial hypertension. BMJ 2003; 326: 641-2

[120] Taylor DN, Pitarangsi C, Echeverria P, Diniega BM. Campylobacter enteritis during doxycycline prophylaxis for malaria in Thailand. Lancet 1988; 2: 578-9.

[121] Arthur JD, Echeverria P, Shanks GD, Karwacki J, Bodhidatta L, Brown JE. A comparative study of gastrointestinal infections in United States soldiers receiving doxycycline or mefloquine for malaria prophylaxis. Am J Trop Med Hyg 1990; 43: 608-13.

[122] Lesens O, Haus-Cheymol R, Dubrous P, et al. Methicillin-susceptible, doxycycline-resistant Staphylococcus aureus, Cote d'Ivoire. Emerg Infect Dis 2007; 13: 488-90.

(C) Briolant et al.; Licensee Bentham Open.

This is an open access article licensed under the terms of the Creative Commons Attribution Non-Commercial License (http://creativecommons.org/licenses/ by-nc/3.0/) which permits unrestricted, non-commercial use, distribution and reproduction in any medium, provided the work is properly cited. 